\title{
Quantitative patterns of motor cortex proteinopathy across ALS genotypes
}

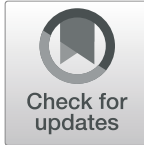

\author{
Matthew Nolan', Connor Scott ${ }^{1}$, Menuka Pallebage Gamarallage ${ }^{1}$, Daniel Lunn², Kilda Carpenter ${ }^{1}$, \\ Elizabeth McDonough ${ }^{3}$, Dan Meyer ${ }^{3}$, Sireesha Kaanumalle ${ }^{3}$, Alberto Santamaria-Pang ${ }^{3}$, Martin R. Turner ${ }^{1}$, \\ Kevin Talbot ${ }^{1}$ and Olaf Ansorge ${ }^{1 *}$ (D)
}

\begin{abstract}
Degeneration of the primary motor cortex is a defining feature of amyotrophic lateral sclerosis (ALS), which is associated with the accumulation of microscopic protein aggregates in neurons and glia. However, little is known about the quantitative burden and pattern of motor cortex proteinopathies across ALS genotypes. We combined quantitative digital image analysis with multi-level generalized linear modelling in an independent cohort of $82 \mathrm{ALS}$ cases to explore the relationship between genotype, total proteinopathy load and cellular vulnerability to aggregate formation. Primary motor cortex phosphorylated (p)TDP-43 burden and microglial activation were more severe in sporadic ALS-TDP disease than C9-ALS. Oligodendroglial pTDP-43 pathology was a defining feature of ALS-TDP in sporadic ALS, C9-ALS and ALS with OPTN, HNRNPA1 or TARDBP mutations. ALS-FUS and ALS-SOD1 showed less cortical proteinopathy in relation to spinal cord pathology than ALS-TDP, where pathology was more evenly spread across the motor cortex-spinal cord axis. Neuronal pTDP-43 aggregates were rare in GAD67+ and Parvalbumin+ inhibitory interneurons, consistent with predominant accumulation in excitatory neurons. Finally, we show that cortical microglia, but not astrocytes, contain pTDP-43. Our findings suggest divergent quantitative, genotypespecific vulnerability of the ALS primary motor cortex to proteinopathies, which may have implications for our understanding of disease pathogenesis and the development of genotype-specific therapies.
\end{abstract}

Keywords: ALS, FTD, Selective vulnerability, TDP-43, C9ORF72

\section{Introduction}

The term 'selective vulnerability' describes the differential susceptibility of cells or anatomically defined systems to disease pathomechanisms. In the context of neurodegenerative disease, this can be further delineated as vulnerability to cellular proteinopathy or vulnerability to degeneration itself; a relationship which cannot be assumed to be linear, as microscopically visible aggregate formation may be an indicator of a successful cellular response to proteotoxicity, dependent on cell-specific concentration-dependent thresholds for protein

\footnotetext{
* Correspondence: olaf.ansorge@ndcn.ox.ac.uk

${ }^{1}$ Nuffield Department of Clinical Neurosciences, University of Oxford, Level 1, West Wing, John Radcliffe Hospital, Oxford OX3 9DU, UK

Full list of author information is available at the end of the article
}

precipitation $[3,16,28,73]$. In amyotrophic lateral sclerosis (ALS), this selectivity is particularly apparent as functionally distinct motor neuron subtypes in close anatomical proximity are variably affected $[13,56]$, however whether this reflects cell-intrinsic vulnerabilities or connectivity, or both, remains unclear. Around $10 \%$ of ALS cases are caused by autosomal dominant mutations of varying penetrance to one or more known genes including C9ORF72 (C9-ALS) [17, 64], SOD1 (ALS-SOD1) [68], FUS (ALS-FUS) [43] and TARDBP [81], with some mutations appearing to predispose patients towards an upper (UMN) or lower (LMN) motor neuron predominant phenotype $[29,60,65,79]$. The primary neuropathological observation in $\sim 95 \%$ ALS cases is the cytoplasmic mislocalization and aggregation of hyper- 
phosphorylated TDP-43 (pTDP-43) within neurons and glia (ALS-TDP) [54].

While ALS patients commonly present with a combination of LMN and pyramidal signs, the nature of disease initiation and progression between the spinal cord and cortex remains unclear. In the 'dying forward' hypothesis, motor cortex dysfunction associated with anterograde glutamate-mediated excitotoxicity precedes LMN dysfunction [19, 24]. However, attempts to identify a histological correlate for this hypothesis using human tissue have produced conflicting results [30, 48, 55], compounded by neuropathological assessment often being confined to subjective readouts of disease burden which are a source of bias and potentially increase type I error. Digital image analysis algorithms have previously been used to accurately quantify TDP-43 pathology more objectively $[35,86]$, and the combination of quantitative disease indicators and genetic architecture can be used to produce neuropathological endophenotypes independent of relatively crude clinical readouts, which may not be up-to-date during the terminal phase of the illness $[2,18,53]$.

Surprisingly, there have been no systematic studies attempting to define and quantify the total proteinopathy burden and its cellular pattern in the primary motor cortex across ALS genotypes since the discovery of the main ALS driver genes and their protein products. We hypothesized that the ratio of motor cortex to spinal cord proteinopathy burden is not uniform across genotypes and that not all cell types are equally affected by protein aggregates. For example, we therefore sought to clarify if inhibitory interneurons, excitatory pyramidal neurons, or oligo-/astroglia preferentially accumulate protein aggregates in the motor cortex. Here, we use quantitative digital image analysis in conjunction with multilevel statistical modelling to show that primary motor cortex phosphorylated (p)TDP-43 burden and microglial activation was more severe in sporadic ALSTDP disease than C9-ALS, that oligodendroglial pTDP43 pathology was a defining feature of all genetic subgroups of ALS-TDP, that ALS-FUS and ALS-SOD1 show less cortical proteinopathy in relation to spinal cord pathology than ALS-TDP, and that neuronal pTDP-43 aggregates are rare in GAD67+ inhibitory interneurons consistent with predominant accumulation in excitatory neurons. Our data from an independent ALS cohort thus contribute to our understanding of motor cortex neuropathology across diverse ALS genotypes.

\section{Materials and methods}

\section{Cases}

Cases were included if the primary clinical presentation was ALS as judged by an experienced neurologist with a special interest in ALS. Presentation with frontotemporal dementia (FTD) was an exclusion criterion. A further inclusion criterion was availability of definitive primary motor cortex (defined by the presence of Betz cells) and lumbar spinal cord blocks. Post-mortem human brain tissue in the form of $10 \mu \mathrm{m}$ sections was obtained from the Oxford Brain Bank, the MRC London Neurodegenerative Diseases Brain Bank and the Sheffield Brain Tissue Bank. Consent and ethical approval for the use of tissue was provided by the generic REC approval of each research tissue bank (Oxford - 15/SC/0639, MRC London - 08/MRE09/38, Sheffield - 08/MRE00/13). All cases were diagnosed post-mortem by an experienced neuropathologist and confirmed genetically via Sanger or whole-exome sequencing. For the purposes of this study, we therefore use the term 'sporadic' to refer to cases with no high-penetrance mutations in known ALS genes and an absence of characteristic genetically-linked pathology. Whole-exome sequencing failed to find a causative mutation in one patient (case 8), but was neuropathologically confirmed as ALS-FUS through the presence of characteristic FUS pathology. Case 6 also exhibited a Y374X TARDBP variant which is predicted to be damaging, but displayed no TDP-43 pathology [40] and overall the case more closely resembles the juvenileonset ALS associated with ALS-FUS. Cases caused by CHMP2B, HNRNPA1, OPTN and TARDBP mutations are present in $<1 \%$ ALS patients, and we were therefore unable to obtain a significant number of these genotypes, however they were included because of their potential for interesting comparisons and clarification of their pathotype. Demographic and clinical details (where available) of all cases are included in the supplementary material and summarised in Table 1.

\section{Tissue sampling}

$10 \mu \mathrm{m}$ sections containing underlying white matter were cut from archival formalin-fixed paraffin-embedded (FFPE) blocks from the primary motor cortex, corresponding to the 'hand knob' region of the homunculus where available. $5 \mu \mathrm{m}$ sections from the same region were used in multiplexed-immunofluorescence experiments. $10 \mu \mathrm{m}$ sections of FFPE lumbar spinal cord were also cut from each case.

\section{DAB-immunohistochemistry}

De-identified sections from sporadic $(n=19)$, C9ORF72 $(n=16)$, SOD1 $(n=11)$, FUS $(n=9), C H M P 2 B(n=2)$, TARDBP $(n=1), \operatorname{HNRNPA1}(n=1), \operatorname{OPTN}(n=1)$ and control $(n=11)$ cases ('s-IHC cohort') were immunohistochemically stained according to standard protocols. Primary antibody clones (Table 2) were chosen based on specificity demonstrated in protein expression databases (e.g Human Protein Atlas) as well as validation in 
Table 1 Summary of all cases used in this study. See supplementary data for subgroup analyses

\begin{tabular}{|c|c|c|c|c|c|c|c|c|c|c|c|c|c|}
\hline \multirow[t]{2}{*}{ Genotype } & \multirow{2}{*}{$\begin{array}{l}n \\
=\end{array}$} & \multirow{2}{*}{$\begin{array}{l}\text { With } \\
\text { FTD }\end{array}$} & \multicolumn{2}{|c|}{ Sex } & \multicolumn{3}{|c|}{ Age (Years) } & \multicolumn{3}{|c|}{ PMD (Hours) } & \multicolumn{3}{|c|}{ Fixation (Weeks) } \\
\hline & & & $M$ & $\mathrm{~F}$ & Range & Mean & Std. Dev. & Range & Mean & Std. Dev. & Range & Mean & Std. Dev \\
\hline Control & 17 & 0 & 10 & 7 & $36-95$ & 62.5 & 14.0 & $18-73$ & 42.5 & 16.0 & $0.2-32$ & 7.1 & 9.2 \\
\hline Sporadic & 39 & 7 & 21 & 18 & $44-90$ & 65.5 & 11.7 & $24-120$ & 47.7 & 22.5 & $0.2-28$ & 5.9 & 8.5 \\
\hline C9ORF72 & 18 & 5 & 11 & 7 & $42-76$ & 60.6 & 9.4 & $4-96$ & 47.5 & 30.5 & $0.2-17$ & 7.5 & 4.8 \\
\hline$S O D 1^{b}$ & 11 & 0 & 3 & 8 & $34-78$ & 55.9 & 14.7 & $5-96$ & 39.0 & 31.9 & $3-20$ & 11.8 & 5.8 \\
\hline FUS $^{\complement}$ & 9 & 0 & 4 & 5 & $18-64$ & 37.9 & 15.2 & $19-72$ & 47.0 & 19.5 & $2-45$ & 12.6 & 13.7 \\
\hline$C H M P 2 B^{d}$ & 2 & 0 & 1 & 1 & $51-69$ & 60.0 & 12.7 & $12-22$ & 17.0 & 7.1 & $3-4.5$ & 3.8 & 1.1 \\
\hline HNRNPA1 $^{\mathrm{e}}$ & 1 & 0 & 0 & 1 & 62 & 62.0 & - & 48 & 48.0 & - & 12.0 & 12.0 & - \\
\hline OPTN & 1 & 0 & 1 & 0 & 54 & 54.0 & - & 72 & 72.0 & - & 12.0 & 12.0 & - \\
\hline$T A R D B P^{9}$ & 1 & 0 & 1 & 0 & 57 & 57.0 & - & 48 & 48.0 & - & 9.0 & 9.0 & - \\
\hline
\end{tabular}

${ }^{\mathrm{a}}$ C9ORF72 - Chromosome 9 open reading frame $72 ;{ }^{\mathrm{b}} \mathrm{SOD} 1$ - Superoxide dismutase $1 ;{ }^{\mathrm{C}} \mathrm{FUS}$ - Fused-in-sarcoma; ${ }^{\mathrm{d}} \mathrm{CHMP} 2 \mathrm{~B}$ - Charged multivesicular body protein $2 \mathrm{~B} ;{ }^{\mathrm{e}}$ HNRNPA1 - Heterogeneous nuclear ribonucleoprotein $\mathrm{A} 1 ;{ }^{\mathrm{f}}$ OPTN - Optineurin; ${ }^{\mathrm{g}}$ TARDBP - TAR DNA-binding protein. PMD = post-mortem delay

previous studies. Targets were visualised using HRPconjugated secondary antibodies (Dako Envision+ kit, Agilent, USA), which were incubated for $1 \mathrm{~h}$ at room temperature before detection using $\mathrm{DAB}$ substrate for $10 \mathrm{~min}$. Extensive quality-control experiments were conducted with each antibody prior to batch staining. Specifically, we tested fixation-dependency of the reaction products and qualitatively screened for non-specific staining. The pTDP-43 antibody performed equally well in short- and long-fixed material, with no background staining. In contrast, FUS antibody showed only weak signal in long fixation cases and SOD1 antibody (SEDI) showed very strong non-specific astroglial staining in control tissue and missed some protein aggregates in SOD1 cortex that were clearly visible on HE sections (e.g. hyaline conglomerate inclusions). These antibodies therefore served to support the diagnosis of ALS-FUS and ALS-SOD1 cases respectively, but were not suitable for quantitative digital analysis. We did not have access to anti-dipeptide antibodies that performed robustly across the cohort. However, p62 proved an excellent generic stain for ALS protein aggregates (including

Table 2 Primary antibody clones used in this study

\begin{tabular}{|c|c|c|c|c|c|c|}
\hline \multirow[t]{2}{*}{ Antigen } & \multirow[t]{2}{*}{ Supplier } & \multirow[t]{2}{*}{ Catalogue \# } & \multirow[t]{2}{*}{ Host } & \multirow[t]{2}{*}{ Clonality } & \multicolumn{2}{|l|}{ Dilution } \\
\hline & & & & & $\overline{\mathrm{HC}}$ & MxIF \\
\hline pTDP-43 ${ }^{a}$ & Cosmo-bio & TIP-PTD-M01 & Mouse & Monoclonal & $1: 40000$ & $1: 10000$ \\
\hline $\mathrm{p} 62^{\mathrm{b}}$ & Abcam & ab91526 & Rabbit & Polyclonal & $1: 1000$ & - \\
\hline FUS $^{c}$ & Sigma-Aldrich & HPA008784 & Rabbit & Polyclonal & 1:300 & - \\
\hline $\mathrm{SOD} 1^{\mathrm{d}}$ & StressMarq Bio & SPC-206 & Rabbit & Polyclonal & $1: 1000$ & - \\
\hline $\mathrm{HuC} / \mathrm{D}^{\mathrm{e}}$ & Santa Cruz & sc-28,299 & Mouse & Monoclonal & - & $1: 400$ \\
\hline SMl312 & Sternberger monoclonals & 837,904 & Mouse & Monoclonal & $1: 2000$ & - \\
\hline NeuN & Abcam & ab177487 & Rabbit & Monoclonal & $1: 2000$ & - \\
\hline Parvalbumin & Swant & 235 & Mouse & Monoclonal & $1: 7500$ & $1: 5000$ \\
\hline GFAPg & Sigma-Aldrich & C9205 & Mouse & Monoclonal & - & $1: 800$ \\
\hline Olig2 ${ }^{h}$ & Abcam & ab109186 & Rabbit & Monoclonal & $1: 400$ & - \\
\hline $\mathrm{TPPP} / \mathrm{p} 25^{\mathrm{i}}$ & Win-Fest Ltd & - & Mouse & Monoclonal & $1: 2000$ & - \\
\hline$|\mathrm{ba}|^{\mathrm{j}}$ & WAKO & 019-19,741 & Rabbit & Polyclonal & - & $1: 400$ \\
\hline \multirow[t]{2}{*}{ CD68 ${ }^{k}$} & Dako & M0876 & Mouse & Monoclonal & $1: 200$ & - \\
\hline & ThermoFisher & MS397 & Mouse & Monoclonal & - & $1: 800$ \\
\hline Collagen IV & Merck Millipore & MAB3326 & Mouse & Monoclonal & - & $1: 4000$ \\
\hline OPTN' & Proteintech & 10,837-1-AP & Rabbit & Polyclonal & $1: 3000$ & - \\
\hline $\mathrm{GAD} 7^{\mathrm{m}}$ & Merck Millipore & MAB5406 & Rabbit & Monoclonal & $1: 1000$ & - \\
\hline
\end{tabular}

${ }^{a}$ pTDP-43 - Phosphorylated transactive DNA response protein 43 kDa; ${ }^{b}$ p62 - Sequestosome; ${ }^{\mathrm{c}} \mathrm{FUS}$ - Fused-in-sarcoma; ${ }^{\mathrm{d}} \mathrm{SOD} 1$ - Superoxide dismutase 1;

${ }^{\mathrm{e}} \mathrm{HuC/D}$ - ELAV-like protein 4; $\mathrm{f}^{\mathrm{S}} \mathrm{SMI} 312$ - Panaxonal Neurofilament marker; ${ }^{\mathrm{g}} \mathrm{GFAP}$ - Glial fibrillary acidic protein; ${ }^{\mathrm{h}}$ Olig2 - Oligodendrocyte transcription factor 2;

'TPPP - Tubulin polymerization-promoting protein; ${ }^{j}$ lba1 - Ionized calcium-binding adapter molecule 1 ; ${ }^{\mathrm{k}} \mathrm{CD} 68$ - Cluster of differentiation 68 ; ' OPTN - Optineurin;

m GAD67 - Glutamate decarboxylase 67 
dipeptides) and was used as a read-out for panaggregation load in the selected ROIs [1, 23, 42]. None of the primary motor cortices contained tau, alphasynuclein or amyloid-beta aggregates. For chromogenic double IHC, pTDP-43 and either GAD67, Olig2 or NeuN primary antibodies (Table 2) were applied to a subsection of the cohort (control $n=3$, ALS $n=7$; Supplementary material). These were sequentially stained using pTDP-43 primary antibody with HRP-DAB detection, followed by either GAD67, Olig2 or NeuN primary antibody and goat anti-rabbit secondary antibody conjugated to alkaline phosphatase (1:1000, Dako, cat\#

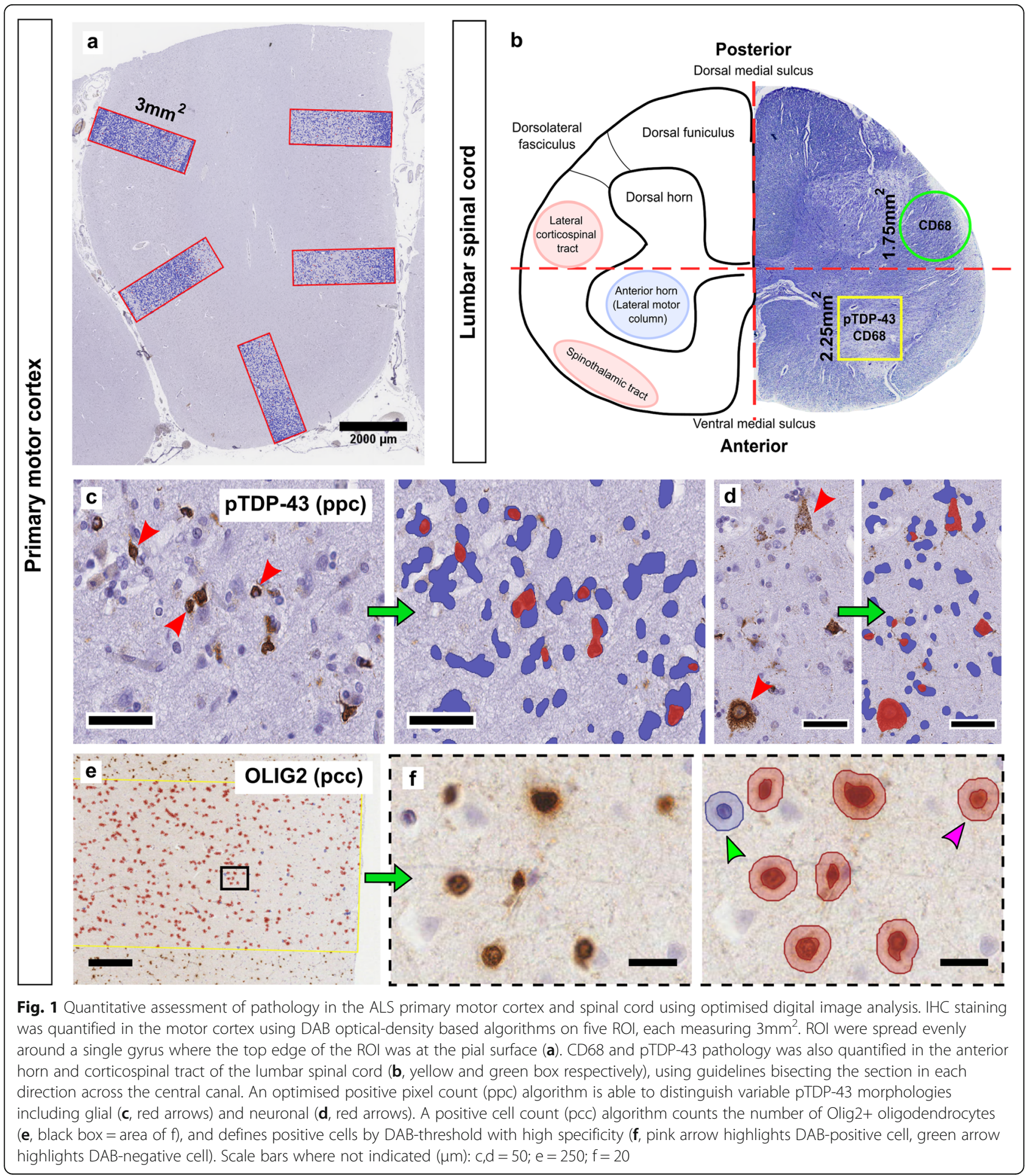


D0487) with FastRed substrate detection. To assess the relative extent of upper versus lower motor neuron involvement, an additional three sections from the lumbar spinal cord region were cut at $10 \mu \mathrm{m}$ from each case and stained with pTDP-43, CD68 and SMI-312. Positive and negative controls were included for each antibody. No staining was seen when the primary antibody was omitted. Counterstaining was performed using Coles haematoxylin. Giant pyramidal cells of Betz define the human primary motor cortex. For all cases therefore, an additional section was stained using haematoxylin and eosin (H\&E) to confirm the presence of Betz cells in layer Vb. Stained sections were then dehydrated, cleared and mounted (Histomount, Thermo-Fisher Scientific, Germany) and viewed using a Zeiss Primo Star microscope (Zeiss, Oberkochen, Germany).

\section{Quantification of single-immunohistochemistry}

Slides were digitally scanned using the Aperio ScanScope AT Turbo system (Leica Biosystems, Germany) and analysed using QuPath software [4]. For pathological quantification of the primary motor cortex, regions of interest (ROI) measuring $1000 \mu \mathrm{m} \times 3000 \mu \mathrm{m}$ were defined and quantified using thresholding and algorithms optimized for each stain. Optimisation of algorithms included adjustment for nuclear/cytoplasmic area, fragmentation of nuclei, pixel size and DAB thresholding. The algorithms themselves are included in the supplementary material. CD68, TPPP/p25 and Olig2 were quantified using a positive cell count (pcc; Fig. 1e-f). Because of the morphological heterogeneity of pTDP-43 and p62 pathology, these were quantified using a positive pixel count (ppc) tool (Fig. 1c,d). Positive pixel count approaches have been used and validated in other human post-mortem studies [35, 86]. Both methods measured the number of positive cells/pixels above a defined DAB optical density (OD) threshold, which was set by plotting mean DAB OD against count for all detections in a given ROI and selecting the lowest OD required to produce minimal false positive/negatives. After optimisation, algorithms were applied consistently throughout. Five ROI were assessed per slide, and placed where the short edge of each ROI was touching the pial surface whilst parallel with underlying white matter. ROI were placed evenly around a single gyrus on each slide, and measurements averaged across the five ROI (Fig. 1a). For CD68, three additional ROI within the subcortical white matter of a single gyrus were also defined and measurements averaged. For lumbar spinal cord sections, one circular ROI measuring $1.75 \mathrm{~mm}^{2}$ and one square ROI measuring $2.25 \mathrm{~mm}^{2}$ was placed in the lateral corticospinal tract region and anterior horn region respectively, on each side of a single lumbar spinal cord slice (Fig. 1b) and measurements averaged. CD68 and pTDP-43 were quantified in the spinal cord using the same algorithms as the motor cortex. The area of 10 anterior horn neurons (5 from each half of the spinal column) from the lumbar region of each case were measured by drawing around the perimeter of each neuron at $\times 40$ mag. Measurements were then averaged and $\log$ transformed for statistical testing.

For oligodendrocyte analysis, criteria for designation as an oligodendrocyte were presence of characteristic round nucleus, chromatin structure and nucleolus, thin rim or perinuclear clear cytoplasm and position as grey matter satellite or subcortical white matter oligodendrocyte. Oligodendroglial TDP-43 inclusions have a characteristic 'comma' or 'wisp-like' appearance. Neuronal criteria were size $(>10 \mu \mathrm{m})$ and shape (triangular or oval), position in cortical layer, presence of peripheral Nissl substance, and a round nucleus with pale homogenous nucleoplasm and a single dark nucleolus.

All analyses were conducted blind to case details including genotype and other IHC results.

\section{Multiplexed immunofluorescence}

Multiplex experiments were conducted on a subset of cases, all with a short $(<48 \mathrm{~h})$ fixation protocol, as most antibodies suitable for multiplex experiments did not work after long ( $>4$ weeks) fixation. Multiplexed immunofluorescence (MxIF) staining and imaging was performed at GE Research using previously described methodologies [25], optimized for short-fixed FFPE neurological specimens. In brief, antibodies (Table 2) were applied two or three at a time across nine sequential staining-and-dye-deactivation rounds to a subsection of our cohort (sporadic $n=18$, C9ORF72 $n=3$, controls $n=5$; Supplementary material). Antigens were detected either by directly fluorophore-conjugated antibodies, by pre-bound fluorophore-labelled antibody binders, or using primary antibody followed by fluorophore-labelled secondary antibodies. The staining of each antibody was imaged on separate channels after each staining round, and DAPI imaging of nuclei was collected in all rounds. Images were collected on custom Olympus IX81 inverted microscopes (Olympus, Tokyo, Japan) using 20x objectives, with multiround image acquisition driven by automated, custom software. Images from different staining rounds were coregistered using DAPI staining (US Patent no. US8369600B2, 2013) [15] and then autofluorescence signal was removed by subtracting an earlier, unstained image from each corresponding stained image [84]. ROIs for MxIF acquisition were selected manually on the whole-tissue tissue sections to cover from the pial surface to the subcortical white matter of each case. Images were reviewed as a single panel composed of $\sim 15$ stitched fields 
of view per case at the University of Oxford using a custom plugin for ImageJ [71] enabling review of the multichannel MxIF image data.

\section{Statistical analysis}

Statistical analysis and graphing was performed using a combination of GraphPad Prism (California, USA) and multi-level generalized linear modelling (GLM) [52, 83] in RStudio (Boston, USA). The procedure used is analogous to a 3-way ANOVA where each individual Case is a block, Protein is a grouping variable and Genotype is a treatment, but in order to avoid over-parameterisation Case was incorported as a random effect in a 2-level multilevel model; thus within-Case correlation is accounted for. Goodness-of-fit chi-squared test revealed the data was negatively-binomially distributed. If the mean Count is $\mu$, a negative-binomial GLM fits the model:

$\log \mu=\alpha+\sum_{i} \beta_{i}$ Genotype $_{i}+\sum_{j} \beta_{j}$ Protein $_{j}+\sum_{i, j} \beta_{i j}$ Genotype $_{i}:$ Protein $_{j}$

Unpaired Welch's $t$-test was used to test oligodendrocyte pathologies. D'Agostino and Pearson method was used to assess normality prior to testing correlation coefficients. Scatterplots are formatted on a logarithmic base 2 scale and labelled as exponents of the base value. Levels for quoted confidence intervals are set at 95\% and $p$-values in the figures are indicated as follows: $* p<0.05, * p<0.01, * * * 0<0.001, * * * * 0<0001$.

\section{Data availability}

The dataset(s) supporting the conclusions of this article are included within the article and its additional files.

\section{Results}

Pathology of the ALS primary motor cortex is highly variable both within and across the spectrum of ALS genotypes

To assess the relative extent of pathology within the primary motor cortex across ALS genotypes, we performed immunohistochemical staining on our cohort ('s-IHC cohort'; see supplementary material) using antibodies for the common pathological markers pTDP-43, p62, and CD68 (Fig. 2; Table 3).

Morphology and severity of pTDP-43 was highly variable, even within cases of the same genotype. No pTDP43 staining was detected in any of the SOD1, FUS or control cases. A formal goodness-of-fit test of a negative-binomial GLM fit for pTDP-43 staining was satisfactory $\left(\chi^{2}(32)=39.577, p=0.167\right)$. On average, pTDP-43 aggregate burden was moderately but significantly higher in sporadic cases than C9ORF72 disease $(p=0.043)$ (Fig. 2g). Notably, pTDP-43 pathology was relatively sparse across all layers in the single heterozygous TARDBP (M337V) mutation case analysed, but was widespread and severe in homozygous ALSOPTN (R217X), particularly within layer II and V and at the grey/white matter border (Fig. 2a,d; Supp Figure 2).

Microglia are the innate immune cells of the central nervous system and robustly express the transmembrane glycoprotein CD68 in response to inflammatory injury as part of an 'activated' phenotype, a process which is commonly implicated in the pathogenesis of ALS (for review, see: $[49,62])$. CD68 staining was therefore performed to assess the relative levels of microglial activation in both the grey and white matter within the primary motor cortex across our cohort (Fig. 2c, f, i). The relative extent of CD68 staining between the subcortical white matter and grey matter correlated in all genotypes (Supp. Figure 3). However, microglial activation was higher within the subcortical white matter than cortical grey matter in nearly all genotypes analysed (Fig. 2i). Differences in grey matter CD68 staining were significant between genotypes; staining was higher in sporadic cases than C9ORF72 $(p=0.037)$ and SOD1 cases $(p=<0.0001)$. In the subcortical white matter, CD68 expression was significantly higher in sporadic ALS-TDP cases than SOD1 $(p=<0.0001)$ and FUS cases $(p=0.0006)$, and also significantly higher in C9ORF72 cases than SOD1 cases $(p=0.0001)$ (Fig. 2i). Finally, and in contrast to the findings of a previous semiquantitative study [12], grey matter CD68 staining correlated closely with pTDP-43 severity in both sporadic and C9-ALS (Fig. 2k).

p62 is a ubiquitin-binding scaffold protein involved in the degradation of marked cargoes via selective autophagy, and is a known component of pTDP-43immunoreactive inclusions. p62 was highest in the OPTN case. Among other genotypes, p62 was highest in C9ORF72 cases, and lowest in HNRNPA1 (Fig. 2b, e, h). p62 was significantly higher in sporadic than FUS ( $p=$ $0.0004)$ and SOD1 $(p=<0.0001)$ cases but not C9ORF72 disease $(p=0.92)$. We found a strong positive correlation between the extent of p62 and pTDP-43 in sporadic ALS [Pearson $r(16)=0.61, p=0.006$ ] (Fig. 2j), however this relationship was not observed in C9-ALS to the same degree [Pearson $r(14)=0.53, p=0.03$ ] (Fig. 2j), reflecting the existence of p62-positive, pTDP-43negative dipeptide repeat proteins produced as a consequence of the C9ORF72 expansion. In contrast to the pTDP-43-CD68 relationship identified in sporadic and C9-ALS, the extent of p62 aggregation did not correlate with CD68 in ALS-SOD1 and ALS-FUS (Fig. 2l).

Taken together, these results show that on average sporadic ALS is associated with a more pronounced motor cortex pTDP-43 proteinopathy and neuroinflammatory pathological phenotype than C9-ALS, implicate TDP-43 as a driver of microglial activation, and broadly highlight 

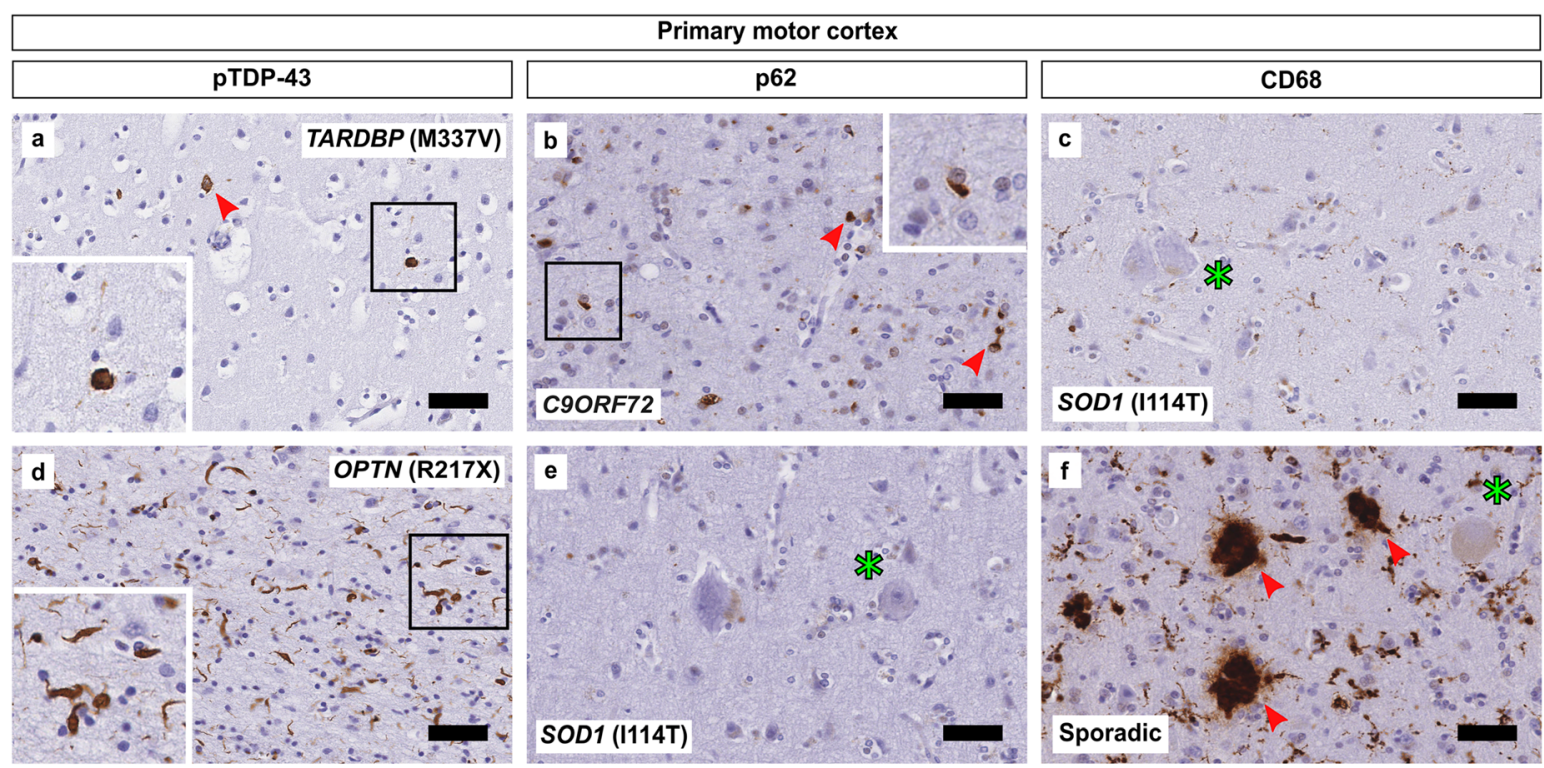

g

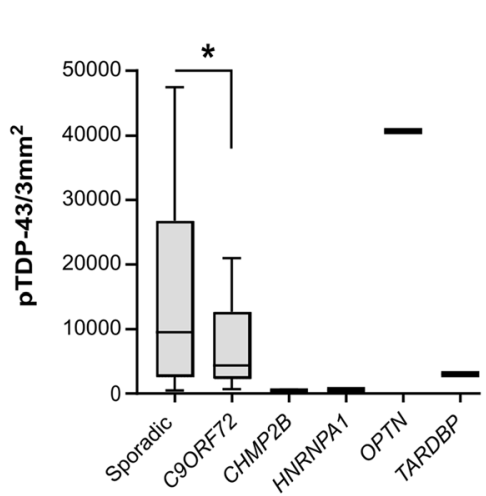

j

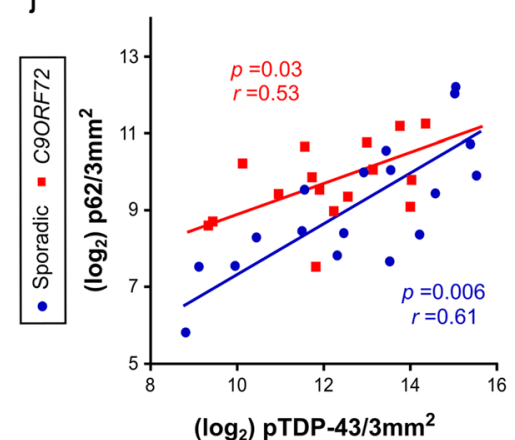

h

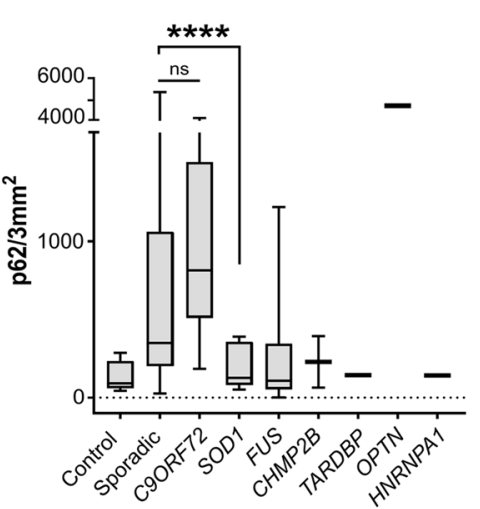

k

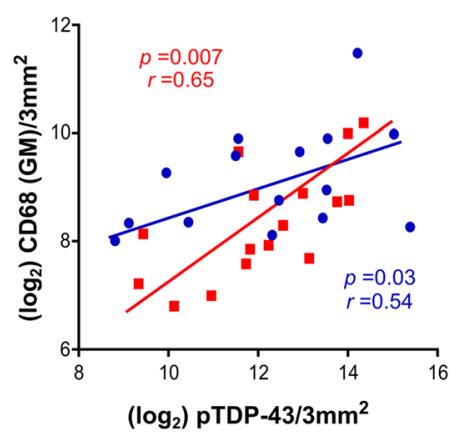

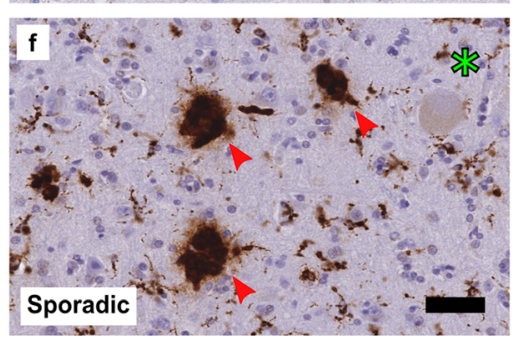

i

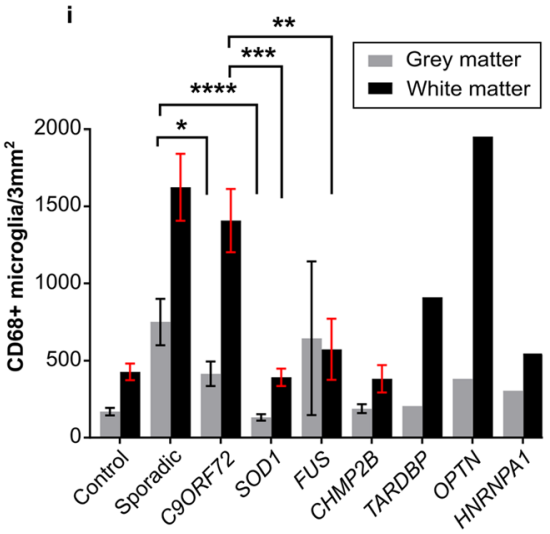

I

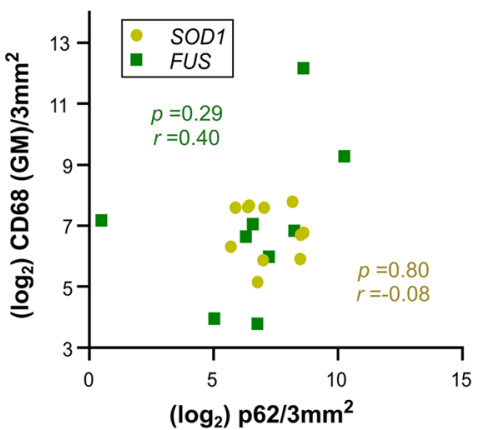

Fig. 2 Pathology of the ALS primary motor cortex is variable both within and across the genotypic spectrum of disease. Relatively little pTDP-43 aggregation was found in a single TARDBP mutation case (a), but this was severe in an OPTN mutation case (d; see also supp. Figure 2). Insets highlight variance of pTDP-43 morphology between genotypes. Average highest pTDP-43 deposition was seen in sporadic cases, which was statistically higher than in C9-ALS $(\mathbf{g})$. Quantification of p62 (b, e, h). Levels of p62 correlated with pTDP-43 in sporadic cases but less so in C9ORF72 disease, reflective of the existence of p62-positive dipeptide repeat protein species unique to C9-ALS (h and $\mathbf{j})$. Cortical microglial activation was highly variable between genotypes (i), and in some cases there was evidence of severe nodular neuronophagia surrounding layer $\checkmark$ neurons ( $\mathbf{f}$. Grey matter CD68 correlated with the extent of pTDP-43 deposition (k) in both sporadic and C90RF72 cases, but this relationship was not recapitulated using p62 and CD68 in SOD1/FUS cases (I). Arrows highlight pathology, asterisks highlight Betz cells. $r$ correlations = Pearson (j) or Spearman $(\mathbf{k}, \mathbf{l})$, results as on figure. Bars in (i) represent means and SEM. Best-fit lines are manually added for illustrative purposes. All scale bars $=50 \mu \mathrm{m}$ 


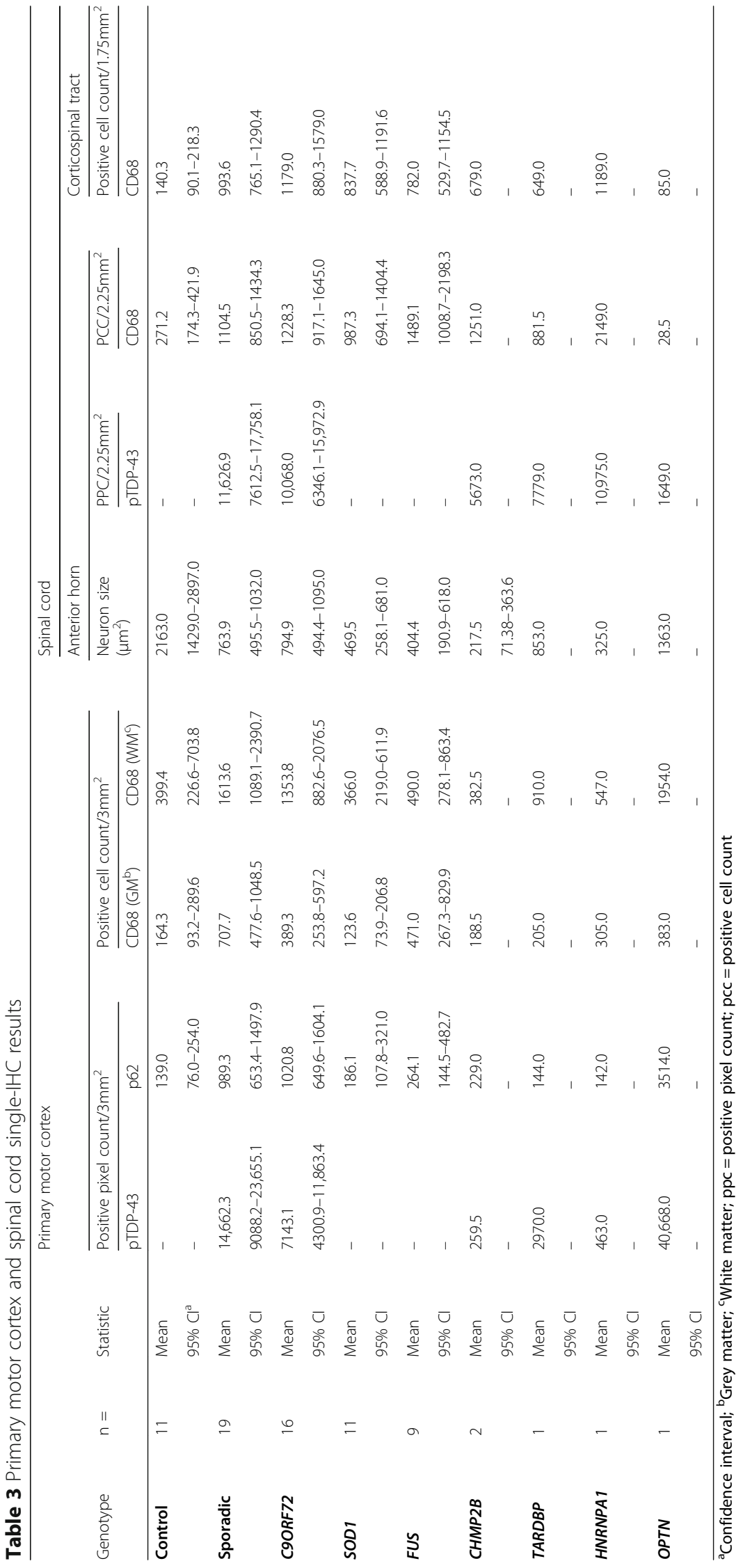




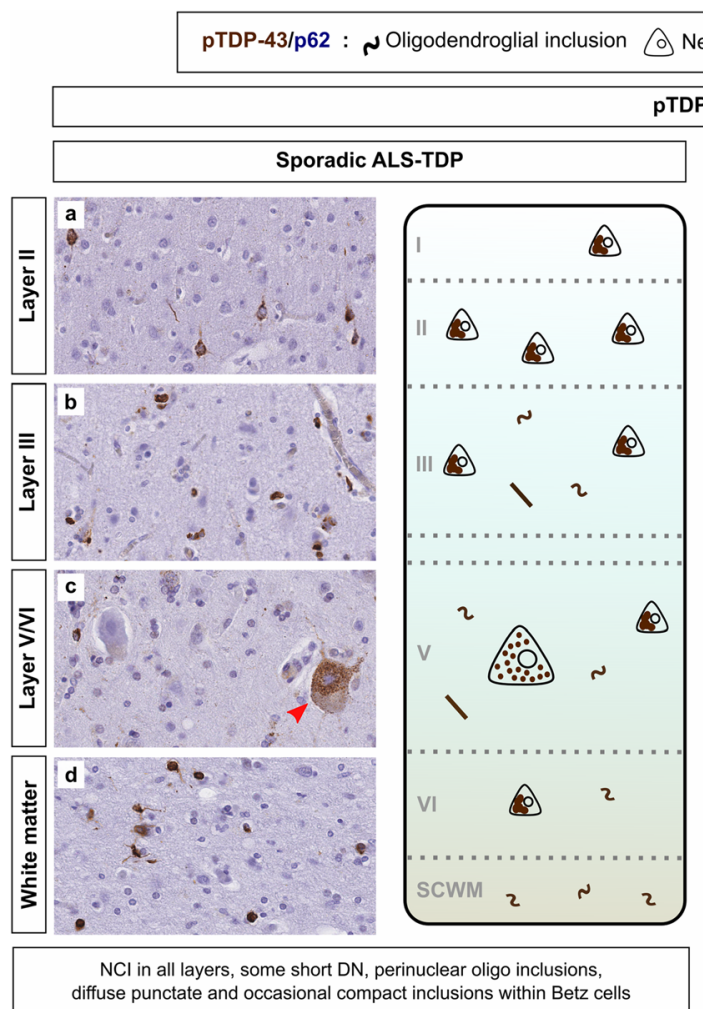

euron

- Grains/punctate \Dystrophic neurite

C9-ALS

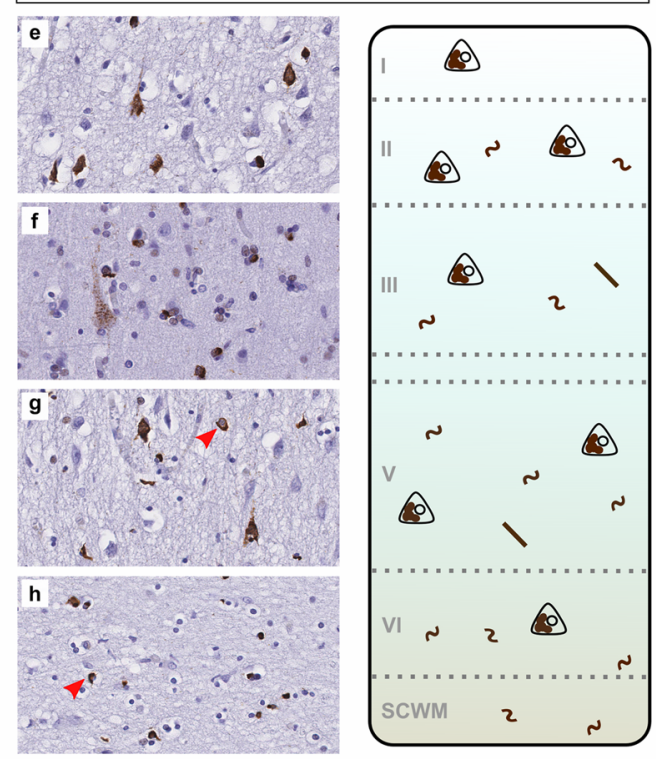

diffuse punclate and occasional compact inclusions within Betz cells

$\mathrm{NCl}$ in all layers, some short DN, prominent perinuclear oligo inclusions, Betz cell pathology less prominent
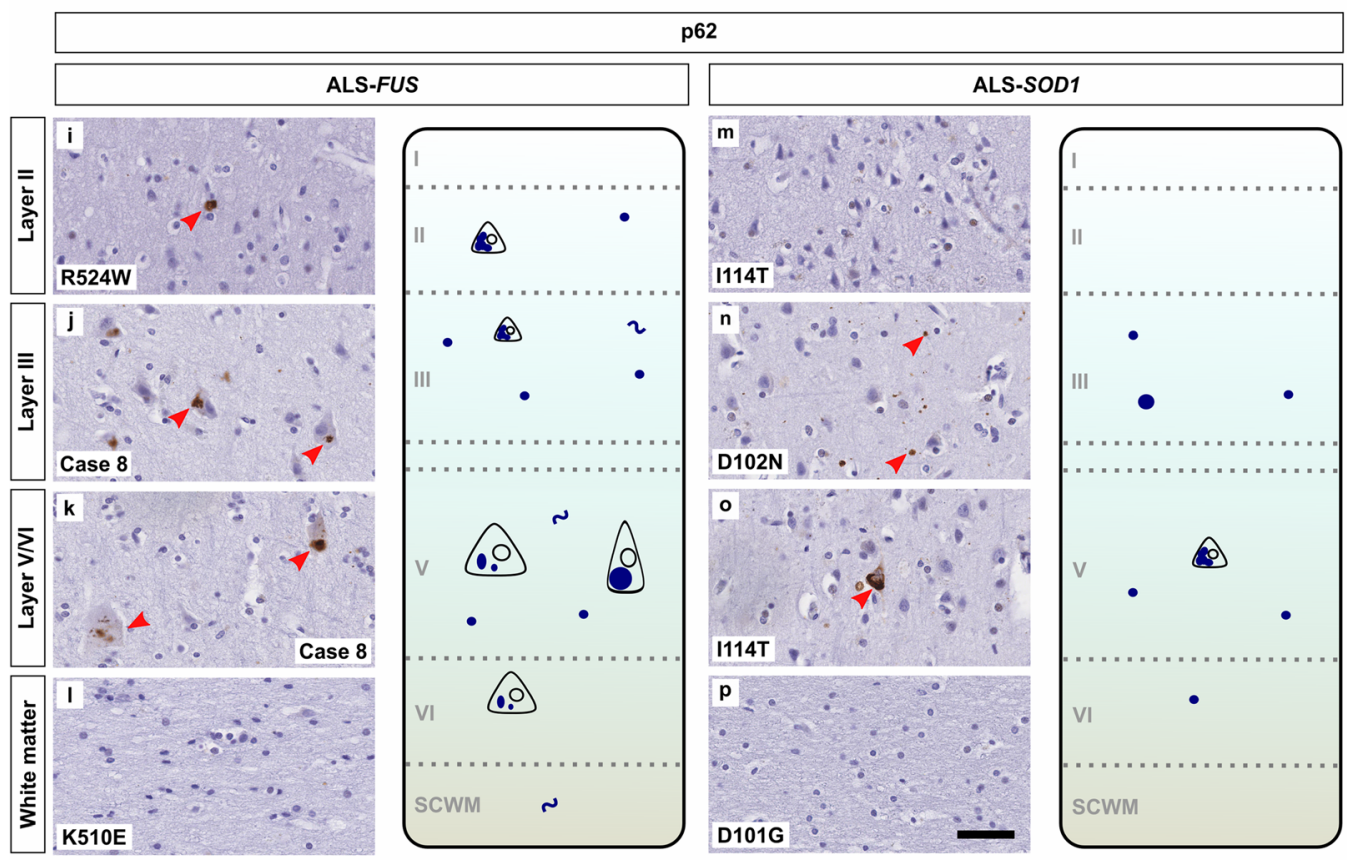

Infrequent, compact $\mathrm{NCl}$ in layers II-VI, variable punctate/grains, rare Betz cell pathology, occasional oligo inclusions

Almost exclusively layers III-VI, variable punctate/grains, occasional $\mathrm{NCl}$, no obvious glial pathology

Fig. 3 Spatial and morphological distribution of proteinopathy across genotypes in the ALS primary motor cortex. Sporadic ALS exhibits NCl and oligodendroglial pathology across layers I-VI as well as the subcortical white matter (a-d). The distribution pattern of pTDP-43 pathology was not entirely dissimilar between sporadic and C9-ALS (e-f), although there was a slight preponderance towards oligodendrocyte inclusions in C9 cases (Fig. $6 \mathrm{~g}$ and h). FUS mutation cases demonstrated infrequent, compact p62-positive $\mathrm{NCl}$ with occasional granular inclusions, as well as occasional oligodendroglial pathology (i-I). SOD1 mutation cases, by contrast, exhibited granular p62 staining confined to the middle cortical layers with infrequent $\mathrm{NCl}$ in layer $\mathrm{V}$, without obvious glial pathology. Arrows highlight respective proteinopathy. Scale bar applicable to all panels $=50 \mu \mathrm{m}$ 
the significant variability in the severity of motor cortical pathology across the spectrum of ALS genotypes.

\section{Spatial and morphological distribution of motor cortical proteinopathy}

TDP-43 proteinopathy is the major neuropathological finding in sporadic and C9-ALS cases, while ALS-FUS and ALS-SOD1 cases are marked by p62-positive FUS and SOD1 aggregates, respectively $[14,51,54]$. Previous studies have described distribution patterns of cortical pTDP-43 pathology in sporadic ALS [77] and FTLD [47], but this has not been examined comparatively between genotypes in ALS. Given the significant variation in the extent of identified pathology between genotypes, we next qualitatively assessed the relative laminar distribution and morphologies of pTDP-43 and p62 proteinopathy in our s-IHC cohort (Fig. 3; for cases assessed see supplementary material).

While the extent was highly variable, the dominant pattern of cortical pTDP-43 pathology in sporadic cases consisted of granular, skein or compact neuronal aggregates, with occasional dot and short thread shaped dystrophic neurites, as well as 'comma' or 'wisp'-like perinuclear oligodendroglial inclusions. Staining was mainly present within layers II-VI (Fig. 3a-c) but all ALS-TDP cases assessed exhibited at least one obvious example of oligodendroglial pTDP-43 pathology within the cortical/subcortical white matter, even in cases with the lowest average grey matter pTDP-43 overall (Fig. 3d). There was occasional, diffuse cytoplasmic punctate pathology within the giant pyramidal cells of Betz (Fig. 3c); compacted aggregates were rare. A harmonized subtyping system exists to describe the heterogeneity of pTDP-43 pathology in FTLD-TDP, based on the relative prevalence and distribution of dystrophic neurites (DN) and neuronal cytoplasmic inclusions (NCI). Assessment of primary motor cortex is not included in this system and it has been mostly applied to patients presenting initially with FTD. It is, however, recognised that cases of FTLD-ALS-TDP are most commonly associated with FTLD-TDP Type B neuropathology, both in sporadic as well as C9-FTLDTDP cases [47]. Although motor cortex pTDP-43 morphology does not appear to be significantly different between FTLD-TDP and FTLD-ALS-TDP cases [78], for clarity, only C9-ALS cases with no clinical history of concomitant FTD were assessed here. Staining was similar in distribution to sporadic cases, with a slight preponderance towards oligodendroglial inclusions (Fig. 3e-h, Fig. $6 \mathrm{~g}$ and h). However, Betz cell pathology was less prominent. No intranuclear pTDP-43-ir inclusions were seen in any of the sporadic or C9-ALS cases examined.

The cellular pattern of proteinopathy in our ALS-FUS and ALS-SOD1 cases was largely consistent with smaller case series described previously [31, 46]. ALS-FUS cases showed predominantly neuronal p62 aggregation within layers I-V in the form of sparse-moderate $\mathrm{NCI}$ and $\mathrm{DN}$ (Fig. 3i, j), although occasional oligodendroglial inclusions were seen. We found rare examples of p62-positive NCI within Betz cells in one of nine (case 8) ALS-FUS cases assessed (Fig. 3k). There were also occasional p62-positive aggregates within the subcortical white matter immediately below layer VI, but these did not reach the deeper white matter. By contrast, p62-positive aggregates in ALS-SOD1 cases were sparse, present almost exclusively within layers III-V, and consisted of variably sized granules (Fig. 3n) with occasional NCI (Fig. 3o). Notably, the majority of p62 aggregations appeared to be concentrated within the middle and deeper layers including layer $\mathrm{V}$, which is the origin of corticospinal projections [50]. No obvious oligodendrocyte pathology was seen. This comparative assessment highlights the morphological variability in which genetic subtypes of ALS manifest as motor cortical disease.

\section{Sporadic ALS-TDP exhibits broad pathological homogeneity across the cortico-spinal neuraxis}

As ALS patients commonly present clinically with a combination of upper and lower motor neuron signs and concomitant pathology, we next performed additional immunohistochemical staining on sections from the lumbar spinal cord region of each case in our singlestain IHC cohort to assess relative pathological predominance in upper or lower motor neuron compartments in our cohort. There was no significant difference in spinal cord pTDP-43 deposition between sporadic and C9ORF72 cases ( $p=0.65$; Fig. $4 \mathrm{~g})$. The lateral corticospinal tract is composed of white matter axons that descend from the cortex and decussate at the level of the medullary pyramids, synapsing with lower motor neurons in the contralateral spinal cord. Although we found a trend for highest CD68 reactivity in the corticospinal tract of C9-ALS cases, this did not reach statistical significance compared with sporadic ALS-TDP cases $(p=$ 0.91; Fig. 3h.) We also found no relationship between pTDP-43 and CD68 in the anterior horn (Fig. 4j).

Staining from the lumbar spinal cord from each case was next compared to its equivalent stain within the primary motor cortex to assess pathological relationships across the corticospinal neuraxis. Similar to a previous semi-quantitative study [11], pTDP-43 severity in the anterior horn correlated with that in the primary motor cortex in sporadic ALS-TDP cases [Pearson $r(17)=0.62$, $p=0.004]$, however this relationship was not present in C9-ALS [Pearson $r(14)=-0.03, p=0.89$ ] (Fig. 4k). CD68 staining within the lateral corticospinal tract correlated with CD68 staining in the primary motor cortex in sporadic [Pearson $r(17)=0.62, p=0.0037$ ] and FUS cases [Pearson $r(7)=0.89, p=0.0013$ ], but not other genotypes $(p>0.2)$ (Fig. 4l). Note that, with 3 out of 4 correlations 


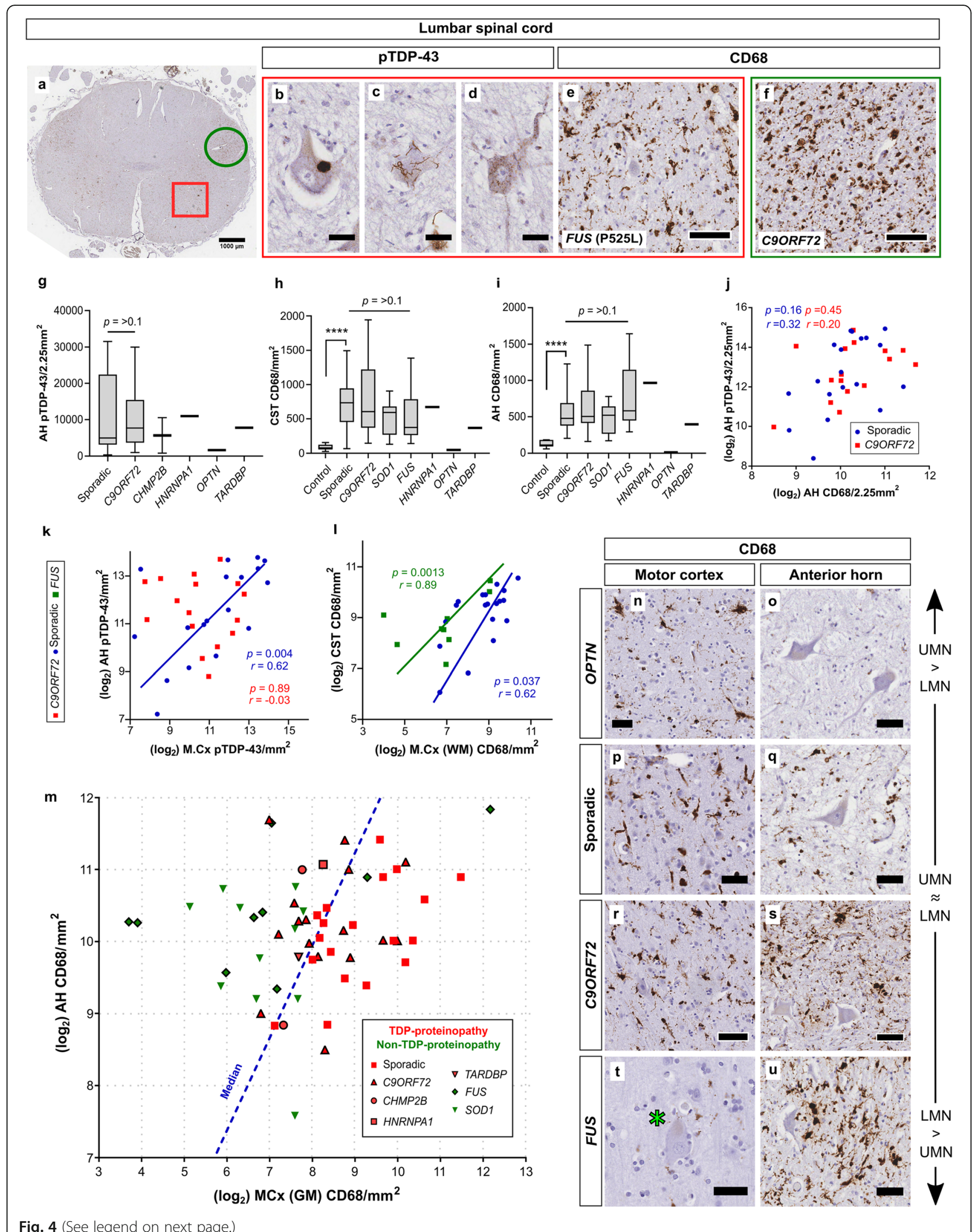

Fig. 4 (See legend on next page.) 
(See figure on previous page.)

Fig. 4 Sporadic ALS-TDP exhibits broad pathological homogeneity across the motor cortex-spinal cord neuraxis. CD68 and pTDP-43 expression was quantified within the anterior horn (a, red box) and corticospinal tract (a, green circle). The morphology of pTDP-43 pathology was varied in anterior horn lower motor neurons, including large discrete inclusions (b), thread-like skeins (c) and diffuse punctate (d). There was no significant difference in pTDP-43 severity between sporadic and C9ORF72 cases (g). CD68 expression (e, $\mathbf{f})$ was variable across ALS genotypes but was not statistically significant $(\mathbf{h}, \mathbf{i})$, and levels of pTDP-43 did not correlate with CD68 expression in the anterior horn (Pearson $\left.r_{;} \mathbf{j}\right)$. pTDP-43 in the motor cortex correlated with pTDP-43 in the anterior horn in sporadic disease but not C9-ALS (Pearson $r ; \mathbf{k}$ ), and CD68 correlated between the corticospinal white matter tract and the subcortical white matter in both FUS and sporadic cases (Pearson $r ; \mathbf{I})$ but not other genotypes ( $p>0.2$ ). CD68 in the lower anterior horn neuron was plotted against grey matter CD68 in the motor cortex $(\mathbf{m})$. This was used to create a predominance ratio for each case that could be used as a covariate in subsequent models, where a lower ratio represents a tendency towards a burden of CD68 in the anterior horn. The dotted blue line in $(\mathbf{m})$ represents the approximate median ratio of our cohort. Therefore, the further each case is from this line, the more extreme the relative UMN/LMN burden of CD68 the case exhibits. Cases are coloured according to whether they are TDPproteinopathies (red) or not (green), showing that non-TDP-proteinopathies are more likely to exhibit a LMN neuropathological predominance when assessed via levels of activated microglia (ALS-OPTN case demonstrated extreme UMN predominance, but is excluded from graph for the clarity of other cases). Representative images of this variation across the neuraxis between genotypes, with an OPTN and FUS mutation representing upper and lower motor neuron spectral extremes, respectively $(\mathbf{n}-\mathbf{u})$. Asterisk in (t) highlights a seemingly normal Betz cell. AH, anterior horn; CST, corticospinal tract; UMN, upper motor neuron; LMN, lower motor neuron. Scale bars where not indicated $(\mu \mathrm{m}): \mathrm{b}, \mathrm{c}, \mathrm{d}=30$, e, $, \mathrm{r}, \mathrm{s}=75$, all others $=50$

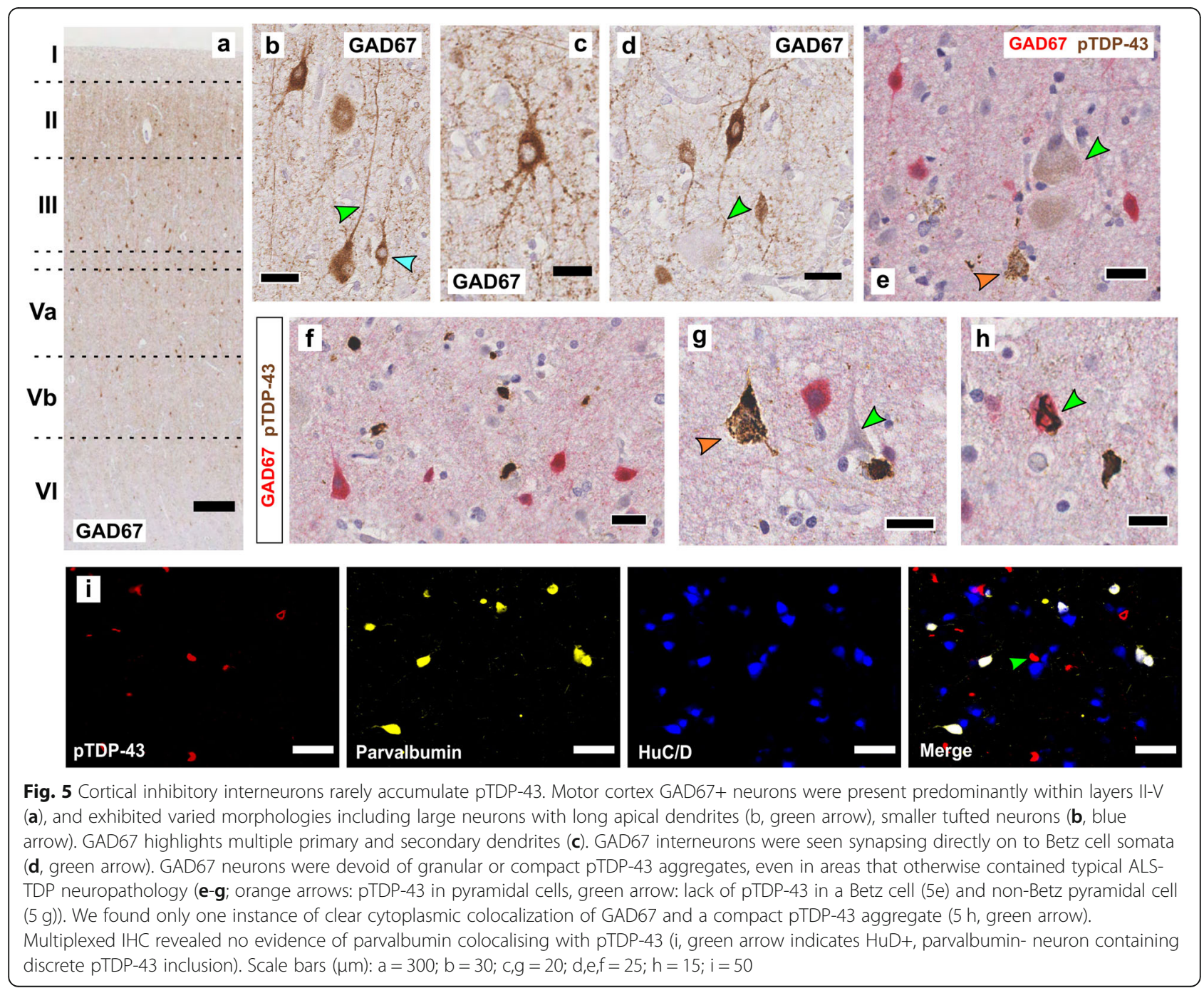



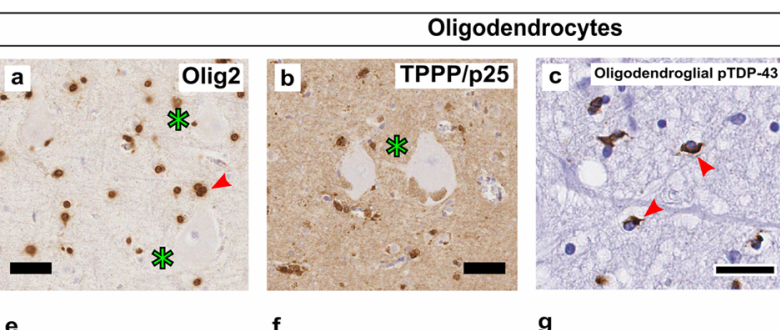

g
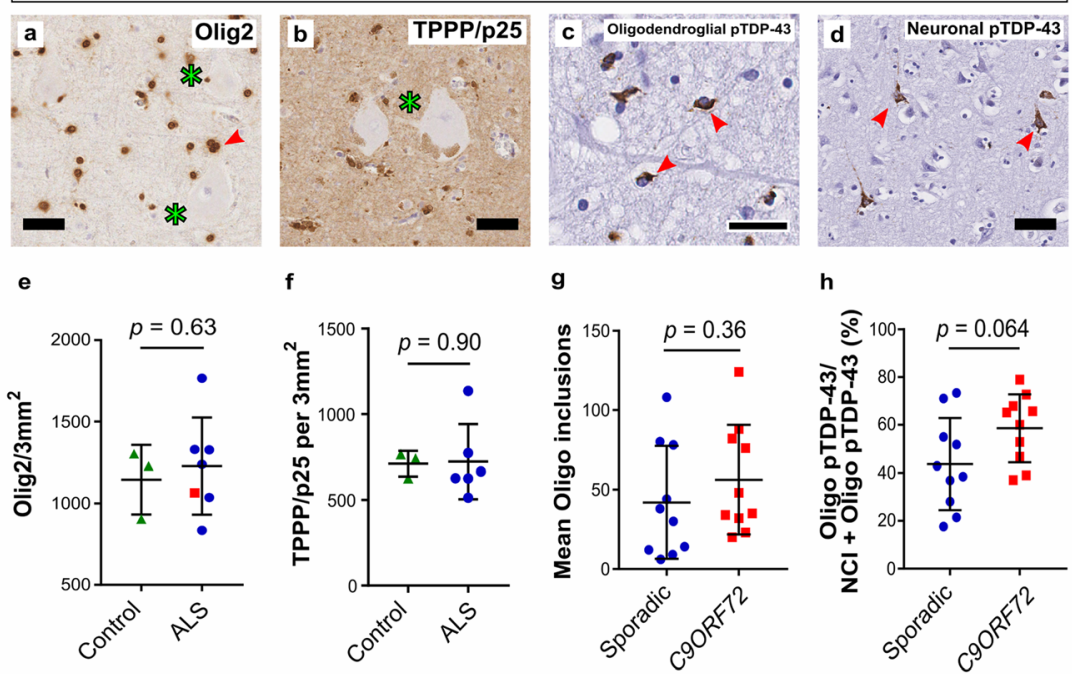

h

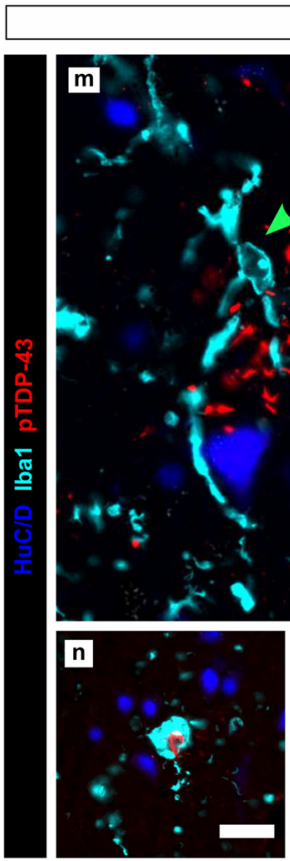

Microglia
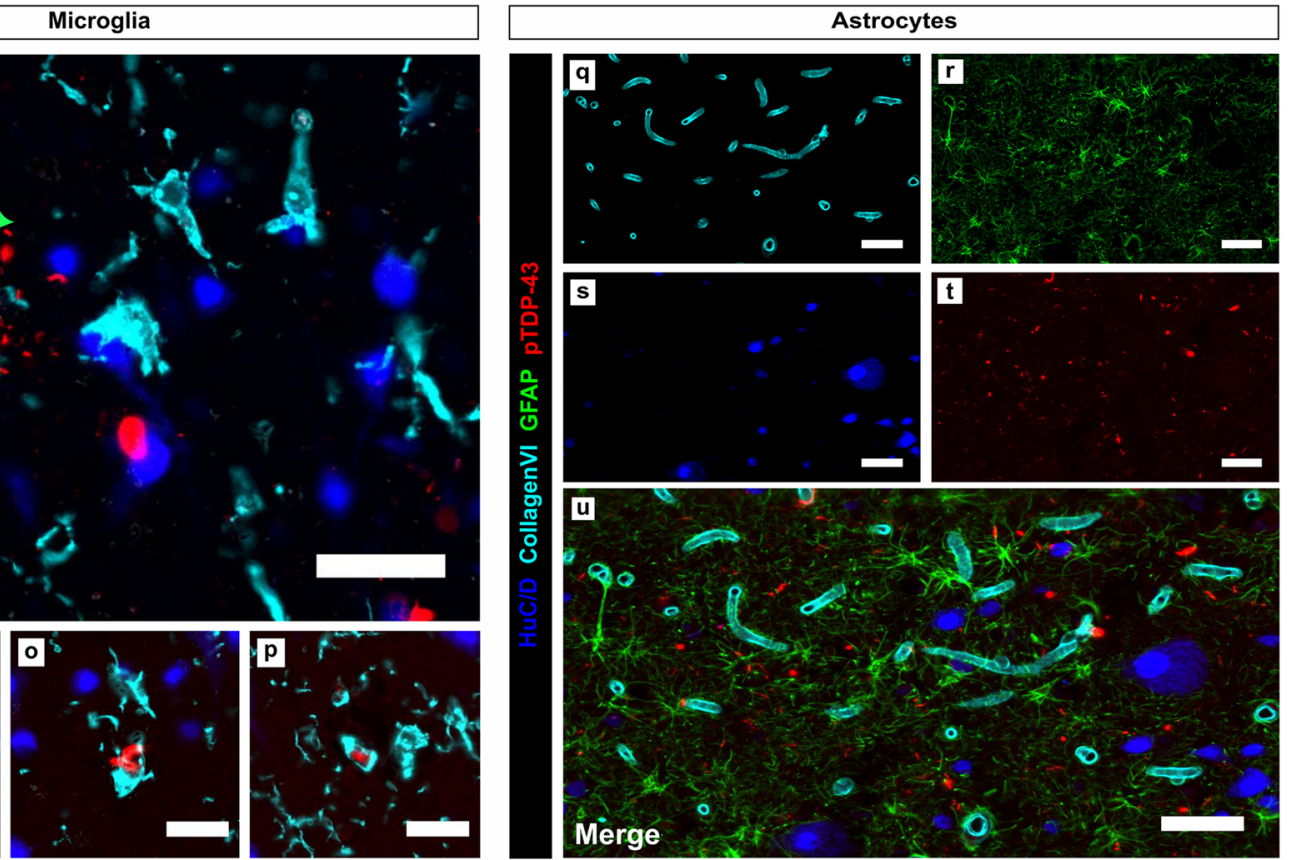

Fig. 6 Differential vulnerability of glial cells in the ALS primary motor cortex. Olig2+ OPC (a) and mature TPPP/p25+ oligodendrocytes (b) were present in all layers of the primary motor cortex. Red arrow in (a) indicates satellite oligodendrocyte surrounding the apical dendrite of a Betz cell (green asterisks). There was no difference in the numbers of mature or immature oligodendrocytes between ALS and control cases (e, f). pTDP-43 inclusions are present in oligodendrocytes (c, red arrows indicated by characteristic 'comma' shaped inclusions) and neurons (d, red arrows indicate neuronal pTDP). Both the mean numbers of oligodendroglial inclusions and the relative predominance of pTDP-43 oligodendroglial pathology was higher in C9-ALS, but was not statistically higher than in sporadic disease (g, h). This relative distribution was confirmed qualitatively using double staining for PTDP-NeuN (i, $\mathbf{j}$ ) and pTDP-Olig2 (k-l, green arrow indicates Olig2-negative neuronal inclusion). Multiplexed immunofluorescence reveals that lba1+ microglia ( $\mathbf{m}-\mathbf{p}$, green arrow in $\mathbf{m}$ indicates $\mathbf{l b a} 1+$ microglia extending processes to surround large layer $\mathbf{V}$ neuron containing pTDP-43 aggregates) occasionally contain pTDP-43, but we could find no clear evidence of this in GFAP+ astrocytes (q-u), even in cases with severe pTDP-43 pathology overall. Bars on graphs $(\mathbf{e}-\mathbf{h})$ represent means and SD. Scale bars $(\mu \mathrm{m}): \mathbf{c}, \mathrm{i}=25, \mathrm{j}=20 ; \mathrm{k}, \mathrm{l}, \mathrm{m}=10$; all others $=50$

being highly significant, simultaneous inference corrections do not modify the conclusions.

A previous semi-quantitative study found a correlation between microglial activation and neuronal loss in both the motor cortex and spinal cord [12]. We therefore next sought to assess the relative predominance of upper/lower motor neuron involvement in our cases by comparing the extent of pathology in the primary motor 
cortex to that in the anterior horn using CD68 as a surrogate marker of neurodegeneration. The extent of CD68 staining between motor cortex and spinal cord was expressed as a ratio, where smaller ratios represent a tendency towards a higher burden of CD68 in the anterior horn (Fig. 4m). Ratios were calculated as:

$$
\frac{\text { M.Cx grey matter } C D 68 / \mathrm{mm}^{2}(\log )}{\text { Anterior horn } C D 68 / \mathrm{mm}^{2}(\log )}
$$

Many ALS-FUS and ALS-SOD1 cases exhibited cortical CD68 comparable to that of controls but showed significant staining within the spinal cord anterior horn (Fig. 4t,u), and $80 \%$ of all non-TDP-43 proteinopathies assessed fell below the calculated median predominance ratio of our cohort (Fig. 4m; Supp Figure 5). The single ALS-OPTN case demonstrated the most significant UMN predominance (Fig. 4n,o; Supp Figure 5). Together, these results show quantitatively that sporadic ALS-TDP exhibits high intraindividual pathological homogeneity across the cortico-spinal neuraxis, and that non-TDP-43 proteinopathies are more likely to display significant lower motor neuron predominance than TDP-43 disease, which is in broad concordance with clinical presentations commonly seen for these genotypes.

\section{GABAergic, GAD67+ interneurons only rarely accumulate pTDP-43 aggregates}

Gamma-aminobutyric acid (GABA) is the main inhibitory neurotransmitter in the brain. It is produced by glutamic acid decarboxylases (GADs) GAD65 and GAD67, of which GAD67 is generally constitutively active and produces $>90 \%$ of basal GABA levels [44]. GAD67 protein expression is a robust generic marker for cell bodies of inhibitory interneurons in human and mouse cortex $[69,72]$. By contrast, no comparatively reliable tool is available as a generic marker for labelling of the soma of human excitatory, glutamatergic neurons, which include extratelencephalic pyramidal tract projection neurons. Therefore, we used GAD67-pTDP-43 double labelling to explore the vulnerability of GABAergic interneurons of the primary motor cortex to the accumulation of pTDP43 aggregates (ALS $n=7$, control $n=3$; supplementary material), with the reasoning that this would allow us to obtain a histological window into potential imbalances of inhibitory and excitatory neurotransmission in ALS motor cortex. In controls, GAD67+ interneurons were mainly present in layers II-V (Fig. 5a), and included large neurons with long apical dendrites (Fig. 5b, green arrow) as well as smaller tufted neurons (Fig. 5b, blue arrow) as well as their dendritic protrusions (Fig. 5c). We also found examples of GAD67+ interneurons directly synapsing on to Betz cells soma (Fig. 5d, green arrow).
Using GAD67-pTDP-43 double labelling in seven ALS cases assessed (see supplementary material for individual cases), we found that GAD67 neurons were devoid of granular or compact pTDP-43 aggregates, even in areas that otherwise contained typical ALS-TDP neuropathology (Fig. 5e-g). We found only one instance of clear cytoplasmic colocalization of GAD67 and a wellformed pTDP-43 aggregate (Fig. 5h), suggesting that pTDP-43 microscopic aggregates only rarely accumulate within interneurons. Additionally, we found no examples of compact pTDP-43 accumulation within Parvalbumin+ interneurons (Fig. 5j), further supporting the idea that pTDP-43 aggregation is more common within excitatory than inhibitory neurons in the ALS primary motor cortex.

\section{Cortical oligodendroglial, but not astroglial, pTDP-43 pathology is a defining feature of ALS-TDP}

The main role of oligodendroglia is the support of neurons, either directly as cortical satellite glia or via the production of insulating myelin. As pTDP-43 accumulation is present within oligodendrocytes and their dysfunction has been implicated in pathogenesis of ALS [61], we sought to assess the numbers of oligodendrocytes within the primary motor cortex as well as the relative abundance of oligodendrocytes containing pTDP-43 inclusions in a subset of our cohort (ALS $n=$ 7 , control $n=3$; see supplementary material for cases used).

Olig2 is a transcription factor highly expressed in immature oligodendrocyte precursor cells (OPC)(Fig. 6a) while Tubulin polymerization-promoting protein (TPPP/ p25) is highly expressed in mature oligodendrocytes (Fig. 6b) [10]. Similar to a previous study in the spinal cord [67], we found no significant difference in mature or immature oligodendrocyte numbers within the ALS-TDP motor cortex grey matter (Fig. 6e and f). We next assessed the prevalence of oligodendrocyte inclusions across our single-IHC cohort. We found at least one obvious instance of oligodendrocyte pTDP-43 inclusion in all ALS-TDP cases assessed (40/40), even in cases that exhibited very low levels of pTDP-43 pathology overall. We next blindly assessed the relative abundance of oligodendrocyte/neuronal pTDP-43 pathology in a randomised selection of ALS-TDP $(n=10)$ and C9-ALS cases $(n=10)$ (Supplementary material). Both the mean number of oligodendrocyte inclusions and the ratio of oligo/neuron inclusions was highest in C9-ALS but neither was significantly higher than in sporadic disease (Fig. $6 \mathrm{~g}$ and $\mathrm{h}$ ). We (OA) qualitatively reviewed all cases with respect to the topography and neuronal vs. oligodendroglial distribution of aggregates. We found that in human motor cortex FUS aggregates involve both neurons and oligodendroglia, but that SOD1 aggregates are 
restricted to neurons. The former assertion is in agreement with previous observations $[5,76]$, however, the latter is based on p62 staining, not staining with confirmation-specific antibody SEDI as this showed strong diffuse astroglial staining even in healthy controls (data not shown); nor did we have access to other conformation-specific SOD1 antibodies.

Microglia may phagocytose inclusions or cells that have been suitably marked for destruction as part of the innate immune system. As microglial activation correlates closely with the extent of pTDP-43 deposition (Fig. 2), we next analysed whether pTDP-43 inclusions could be visualised within microglia themselves. Similar to a previous study [59], we found several examples (Fig. 6mp) of Iba1+ microglia either actively phagocytosing or containing pTDP-43 in several of the TDP-43 proteinopathy cases analysed. Astrocytes are a diverse glial subtype whose roles include mediation of potassium ions across the synapse and the provision of neurotrophic support. However while cortical astrogliosis is a feature of ALS [41], consistent with a previous study [77] there was no obvious perivascular pTDP-43 pathology suggestive of astrogliopathy and using pTDP-43-GFAP double labelling we were unable to find any clear evidence of pTDP-43 aggregates within GFAP+ astrocytes themselves (Fig. 6q-u).

Betz cells are giant pyramidal neurons unique to the primary motor cortex that reside within layer $\mathrm{Vb}$, and can be distinguished histologically by their large size, accumulation of intracellular lipofuscin, and circumferential somatodendritic architecture. Similar to a previous study [9], we observed pTDP-43 accumulation within Betz cell perinuclear cytoplasm only rarely (supp. Figure 6a-d). However, in several cases we did find evidence of severe microgliosis surrounding or seemingly replacing large pyramidal neurons in layer V (supp. Figure 6e), suggesting that processes within these large neurons may induce a selective vulnerability to the microglial response, and indicating active neuronophagia even in the clinical end-stage of the disease (Fig. 2f), a phenomenon which has been previously reported in spinal motor neurons [58].

Together, these results highlight cortical pTDP-43 oligodendrocyte pathology as a defining feature of ALSTDP across all studied genotypes, and suggest a clear neuronal/glial subtype specific vulnerability to proteoaggregation in the ALS primary motor cortex.

\section{Discussion}

We report, to our knowledge, the first large-scale, digital microscopy-guided neuropathological analysis of the burden and pattern of proteinopathies of the primary motor cortex in ALS since the discovery of the main disease defining genotypes. A strength of our study is the combined use of image-analysis algorithms with statistical modelling to allow a comprehensive, fully quantitative approach to pathological assessment. We consider these quantitative traits to be more proximally related to genetic variables and pathobiology than clinical data (e.g. UMN vs. LMN predominance), which were limited, not quantitative and unlikely to be reflective of neuraxis involvement at the end stage of the disease. Importantly, the genetic stratification of our cohort allowed us to begin to assess ALS neuropathological endophenotypes, the utility of which has been demonstrated in other settings, such as frontotemporal lobar degeneration and hippocampal sclerosis $[2,6,26,27$, 53].

Our dataset stems from an independent, previously unpublished cohort of individuals with ALS stratified by genotype. It allows us to contribute several important findings to the field: First, in the group of ALS associated with phosphorylated (p)TDP-43 proteinopathy, sporadic disease was characterised by a higher pTDP burden in the motor cortex than C9-ALS, and all genotypes in this group demonstrated oligodendroglial pTDP-43 inclusion body pathology. Second, overall motor cortex proteinopathy burden of ALS-FUS and ALS-SOD1 is less severe than that of ALS-TDP. Third, inhibitory interneurons appear to be less prone to accumulation of microscopic pTDP-43 aggregates than excitatory neurons.

A key point in discussions concerning selective vulnerability is the distinction between cellular vulnerability to proteinopathy, and vulnerability to degeneration itself. The toxicity of TDP- 43 has been demonstrated in vitro through a range of mechanisms including its effects on endocytosis, autophagy and stress-granule formation [34, 37, 45]. However, the cellular effect of this toxicity in humans is less well understood, and the relative contribution of toxic gain of function and loss of physiological nuclear function of TDP-43 remains difficult to disentangle. We observed high interindividual variation in the extent of pTDP-43 across our cohort, including in patients of the same genotype. pTDP-43 aggregation was amongst the lowest in the single TARDBP mutation case assessed (Fig. 2a,g; supp Figure 2), however notably it has not been formally established that the pattern and/ or severity of pTDP-43 pathology in TARDBP mutation cases is the same as in ALS-TDP or C9-ALS. Understanding the extent of this variation across genotypes is relevant because clearance of pathological cytoplasmic TDP-43 rescues motor deficits and extends lifespan in mice [82], and clinical trials involving immunotherapy are ongoing in humans. Unidentified genetic factors are likely an influence on the overall extent of pathology, however the majority of cases used in this study were sequenced using whole-exome or SNP-array based sequencing using blood-derived DNA, meaning any potential 
CNS-specific variation in the majority of the genome is unaccounted for. In our study, the main genotypes were sporadic disease and C9-ALS; we did not have data on ATXN2 intermediate expansions. In this context it is interesting to note that Yang et al. [85], using immunoblots for pTDP-43, found evidence of higher levels of pTDP-43 in motor cortex of C9-ALS and in spinal cord of ALS-TDP with intermediate ATXN2 expansions. To our knowledge, it has not been formally studied how increased levels of biochemically-determined insoluble pTDP-43 relate to unbiased quantification of lightmicroscopically visible pTDP-43 aggregates in the same anatomical compartment. Our data revealed a significant, but not dramatic difference in microscopic pTDP43 burden in motor cortex of C9-ALS carriers versus sporadic ALS-TDP ( $p=0.043)$; however, we observed a trend towards higher oligodendroglial pTDP-43 burden in C9-ALS.

We detected a differential vulnerability of specific cell types to the aggregation of pTDP-43. This can be summarised as follows: excitatory neurons within the motor cortex are more prone to form pTDP-43 aggregates than inhibitory interneurons, and oligodendroglial, but not astroglial, pTDP-43 pathology should be considered a defining feature of ALS-TDP in cases with and without proven genetic aetiology. We found evidence of oligodendrocyte pathology in every ALS-TDP case assessed in this study, demonstrating cortical oligodendroglial proteinopathy as a defining feature of the disease. However, the mechanisms by which oligodendroglial aggregations potentially cause dysfunction are unclear. While the cellular origin of TDP-43 pathology in humans is not known, studies have suggested that TDP-43 can be transmitted between cells both in culture and in vivo $[20,63]$. It is plausible therefore that pTDP-43 oligodendroglial pathology is preceded by cell-to-cell transmission of seed-competent soluble TDP-43, propagating its non-cell autonomous effects. Notably, and contrasting observations in the spinal cord [21], we found that this pattern of cortical neuronal and oligodendroglial proteinopathy is also a hallmark of ALS-FUS motor cortex, but not of ALS-SOD1. This is unexpected, as the concept of non-neuronal - including oligodendroglial contributions to ALS neurodegeneration has been established in the $S O D 1^{\mathrm{G} 93 \mathrm{~A}}$ mouse model $[36,61]$. Whether this indicates a differential vulnerability of oligodendroglia to RNA-binding protein homeostasis or of different oligodendroglial subtypes remains to be established.

There is no doubt however that microglial activation occurs across all genetic subtypes of human ALS, although whether this response is in itself pathogenic or primarily a subsequent reactive event is debated. Our results show that the extent of this response in the primary motor cortex varies significantly between ALS genotypes, and confirms findings ante mortem concerning the difference in microglial burden between upper and lower motor neurons in sporadic ALS [74, 80]. Several ALS genes have been implicated in the innate immune response specifically; for example C9ORF72 is highly expressed in myeloid cells $[57,66]$, conditional myeloid deletion of OPTN results in dysmyelination and axonopathy [32], and removal of mutant SOD1 from microglia prolongs lifespan in transgenic mice [7]. However, comparably low numbers of cortical activated microglia were seen in SOD1 and FUS cases, which exhibited a relatively low extent of cortical proteinopathy, while the highest microglial response was seen in sporadic and C9ORF72 cases, which on average demonstrated a higher burden of concomitant pTDP-43. This suggests that TDP-43 proteoaggregation specifically determines the extent of the microglial response, rather than the expression of a mutant protein within microglia themselves. We found several examples of pTDP-43 aggregates within microglia (Fig. 6), though we cannot say whether these cells actively phagocytosed the aggregates, or the inclusions arose within the microglia as result of the disease process. We also found no evidence of pTDP-43 inclusions within GFAP+ astroglia, despite cortical astrogliosis being a feature in ALS [41]. Similar to a previous study, we also found only some evidence of compact pTDP-43 aggregates within Betz cell somata [9], although we did find evidence of severe microgliosis and neuronophagia surrounding large layer $\mathrm{Vb}$ neurons in several cases, which has also been previously reported [33]. It has been noted that some ALS Betz cells seem to lose expression of the native protein entirely [9]. This raises interesting questions regarding whether Betz cells are perhaps selectively vulnerable to the toxicity conferred by soluble TDP-43 and its effects on microglia, and, relative to other neurons, whether they lack the capacity to concentrate insoluble TDP-43 in microscopically visible compact inclusions, which may be neuroprotective. In this context it is interesting to note that our data suggest inhibitory interneurons of the primary motor cortex do not seem to form abundant microscopically visible compact pTDP-43 aggregates either. We may, therefore, infer that non-Betz excitatory neurons carry the vast majority of neuronal pTDP-43 aggregate burden in human motor cortex. Whether this reflects cell-intrinsic biochemical traits of GABAergic neurons or is an expression of an inhibitory-excitatory local network imbalance remains unknown. Experimental evidence appears to indicate that excitatory layer $\mathrm{V}$ pyramidal activity is directly associated with the accumulation of ubiquitinated aggregates in TDP-43 mutant mice [87], however it is likely that more complex genotype-dependent interactions between neurons are at play [38]. In any case, our inference that excitatory 
pyramidal neurons, but not inhibitory interneurons, are the primary neuronal cell type accumulating pTDP-43 aggregates is consistent with the hypothesis that pTDP43 propagation through the human nervous system is initiated by them [8].

Our study contains several limitations. We analysed a region of the primary motor cortex corresponding to the hand region of the homunculus wherever possible, however we cannot guarantee that this region was analysed in every case, and it is possible that the expression of proteins described in this paper vary across the somatotopically defined regions of the primary motor cortex. Additionally, we have not stratified our cohort by hemisphere sampled, and it is possible that ALS pathology varies between the same region of different hemispheres, particularly as initial clinical presentation is often lateralised. We note that there is some evidence of asymmetrical TDP-43 pathology in FTLD (without ALS) and Alzheimer's disease [75], but such asymmetry was not found in two cases of ALS-FTLD [39]. The precise variation of ALS pathology across hemispheres is therefore of an unknown significance. We also used CD68 to create a predominance ratio for each case that reflected the comparable levels of involvement between the primary motor cortex and lumbar spinal cord. This was necessary because there is a degree of corticospinal motor neuron loss even in patients that display almost purely LMN signs [70]. Ideally, neuronal loss itself would have been used to create a predominance indicator. However, NeuN, the most commonly used pan-neuronal marker in mouse studies, displays a strong fixation dependency and is unsuitable for use with the kind of archival material used in the single-IHC component of this study, in which we aimed to achieve the highest possible numbers of cases for analysis.

In summary, we used a quantitative immunohistochemical approach to comprehensively analyse pathology within the primary motor cortex in a large cohort of genetically-defined ALS cases. Our results demonstrate a clear genotype-specific vulnerability to ALS proteinopathy, which may provide targets for the design of future therapeutics through genotype-specific amelioration of cortical pathology.

\section{Supplementary information}

Supplementary information accompanies this paper at https://doi.org/10. 1186/s40478-020-00961-2.

Additional file 1 Supp Fig. 1: Additional staining of pathology in the motor cortex and spinal cord. Staining for OPTN was highly positive in all neurons in controls (A), but absent in an OPTN mutation case (D), reflecting the truncation of the protein upstream of the antibody epitope. Asterisks in (B) mark the presence of Betz cells. Arrows in (E) mark mislocalized and aggregated FUS inclusions. Arrows in (F) mark the presence of aggregated SOD1 protein in a case with the SOD1 D101G mutation (case 31). As discussed in the text, antibody SOD1 SPC-206 did highlight solid compact and skein aggregates but had strong background staining $(C)$, making it unsuitable for quantitative automated image analysis. P62 was used instead as a marker for compact SOD-1 associated protein aggregates; granular aggregation of misfolded wild-type SOD1, which has been suggested to be present in all genotypes of ALS, was not revealed by p62 immunohistochemistry [22]. Scale bar applicable to all panels $=50 \mu \mathrm{m}$. Supp Fig. 2: Significant variation in the extent of pTDP-43 pathology between TARDBP and OPTN ALS mutation cases. pTDP-43 pathology in the TARDBP case was sparse, and consisted almost exclusively of compact $\mathrm{NCl}(\mathrm{a}, \mathrm{b}$, red arrows highlight pathology). In contrast, pathology was severe and widespread in the homozygous ALSOPTN case $(c, d)$, with $\mathrm{NCl}$, oligo inclusions and dystrophic neurites in all layers, including the subcortical white matter (e). Supp Fig. 3: CD68 staining between the primary motor cortex (a) and lumbar spinal cord (b) white and grey matter is positively correlated in all the genotypes tested; Pearson $r$, results as on figure. Best fit lines are manually added for illustrative purposes. Supp Fig. 4: Assessment of anterior horn neuron size. Anterior horn degeneration and shrinkage was most prominent in FUS (b) and SOD1 cases, and noticeably less severe in the single ALS-OPTN case (c), however there was significant intraindividual differences within genotypes (d). pTDP-43 aggregation in the anterior horn did not correlate with anterior horn neuron shrinkage/loss in either sporadic (blue dots) or C9ORF72 disease (red squares) (e). Supp Fig. 5: Calculated predominance ratios for single-IHC cohort using CD68 as a surrogate marker of neurodegeneration. Ratios were calculated by dividing the log expression of motor cortex CD68/ $\mathrm{mm}^{2}$ by the log expression of anterior horn CD68/ $\mathrm{mm}^{2}$. Lower ratios therefore represent a higher $L M N$ burden of activated microglia. Supp Fig. 6: Betz cells occasionally display pTDP-43 aggregation but also occasionally nodular microgliosis and neuronophagia. MxIF analysis did not reveal significant evidence of pTDP-43 within Betz cells $(a, b$, green lined arrows indicate unaffected Betz cells), but it can occasionally be seen in some cases $(c, d)$. However we did also find evidence of nodular microgliosis surrounding large layer $V$ neurons in some cases (e). $U L=$ upper layers, $D L=$ deeper layers. Scale bars where not indicated $(\mu \mathrm{m}): \mathrm{a}=40, \mathrm{~b}, \mathrm{e}=50$. Supp Table 3: Numerical results for Olig and TPPP/ p25 quantification.

Additional file 2

\section{Abbreviations}

ALS: Amyotrophic Lateral Sclerosis; FTD: Frontotemporal Dementia;

FTLD: Frontotemporal lobar degeneration; TDP-43: Transactive Response DNA binding protein 43-kDa; FUS: Fused in Sarcoma; OPTN: Optineurin; LMN: Lower motor neuron; UMN: Upper motor neuron; MxIF: Multiplexed immunofluorescence; SOD1: Superoxide dismutase 1; C9ORF72: Chromosome 9 open reading frame 72

\section{Acknowledgments}

We are grateful to the Oxford Brain Bank, Sheffield Brain and Tissue Bank and the MRC London Neurodegenerative Diseases Brain Bank for providing the tissue used in this study. Tissue was obtained from the Sheffield Teaching Hospitals NHS Foundation Trust and the Kings College Hospital NHS Foundation Trust as part of the UK Brain Archive Information Network (BRAIN UK) which is funded by the Medical Research Council and Brain Tumour Research. MN was funded by a PhD studentship from the Motor Neurone Disease Association (grant \# Ansorge/Oct14/977-792). KT receives funding from the Motor Neurone Disease Association, SMA Trust and Medical Research Council. We gratefully acknowledge support by the Motor Neurone Disease Association, the Medical Research Council (MRC), Brains for Dementia Research (BDR) (Alzheimer Society and Alzheimer Research UK) and the NIHR Oxford Biomedical Research Centre. The views expressed are those of the authors and not necessarily those of the NHS, the NIHR or the Department of Health. This work uses data provided by patients and collected by the NHS as part of their care and support and would not have been possible without access to this data. The NIHR recognises and values the role of patient data, securely accessed and stored, both in underpinning and leading to improvements in research and care. We thank the laboratory staff within the Academic Unit of Neuropathology, Oxford, as well as the donors and their families, without whom our research would not be possible. 


\section{Authors' contributions}

MN implemented the study, performed experiments and data analysis and wrote the manuscript. OA conceived the study, analysed data and wrote the manuscript. KC quantified the oligodendrocyte PTDP-43 pathology and SK provided technical expertise and performed the MXIF component. DL provided assistance with statistical analysis, and is now sadly deceased. All authors read, contributed to and approved the final manuscript.

\section{Competing interests}

DM, AS-P, EM and SK are employed by General Electric Company, USA. General Electric did not receive or provide any form of payment for their contribution to the study. The other authors declare no competing interests.

\section{Author details}

'Nuffield Department of Clinical Neurosciences, University of Oxford, Level 1, West Wing, John Radcliffe Hospital, Oxford OX3 9DU, UK. ${ }^{2}$ Department of Statistics, University of Oxford, Oxford, UK. ${ }^{3}$ GE Research, Niskayuna, NY, USA.

Received: 1 June 2020 Accepted: 2 June 2020

Published online: 02 July 2020

\section{References}

1. Al-Sarraj S, King A, Troakes C, Smith B, Maekawa S, Bodi I, Rogelj B, AlChalabi A, Hortobagyi T, Shaw CE (2011) p62 positive, TDP-43 negative, neuronal cytoplasmic and intranuclear inclusions in the cerebellum and hippocampus define the pathology of C9orf72-linked FTLD and MND/ALS. Acta Neuropathol 122:691-702. https://doi.org/10.1007/s00401-011-0911-2

2. Allen $M$, Burgess JD, Ballard T, Serie D, Wang X, Younkin CS, Sun Z, Kouri N, Baheti S, Wang C et al (2016) Gene expression, methylation and neuropathology correlations at progressive supranuclear palsy risk loci. Acta Neuropathol 132:197-211. https://doi.org/10.1007/s00401-016-1576-7

3. Baloh RH (2011) TDP-43: the relationship between protein aggregation and neurodegeneration in amyotrophic lateral sclerosis and frontotemporal lobar degeneration. FEBS J 278:3539-3549. https://doi.org/10.1111/j.17424658.2011.08256.x

4. Bankhead P, Loughrey MB, Fernandez JA, Dombrowski Y, McArt DG, Dunne PD, McQuaid S, Gray RT, Murray LJ, Coleman HG et al (2017) QuPath: open source software for digital pathology image analysis. Sci Rep 7:16878. https://doi.org/10.1038/s41598-017-17204-5

5. Baumer D, Hilton D, Paine SM, Turner MR, Lowe J, Talbot K, Ansorge O (2010) Juvenile ALS with basophilic inclusions is a FUS proteinopathy with FUS mutations. Neurology 75:611-618. https://doi.org/10.1212/WNL. Ob013e3181ed9cde

6. Bennett DA, De Jager PL, Leurgans SE, Schneider JA (2009) Neuropathologic intermediate phenotypes enhance association to Alzheimer susceptibility alleles. Neurology 72:1495-1503. https://doi.org/10.1212/WNL. 0b013e3181a2e87d

7. Boillee S, Yamanaka K, Lobsiger CS, Copeland NG, Jenkins NA, Kassiotis G, Kollias G, Cleveland DW (2006) Onset and progression in inherited ALS determined by motor neurons and microglia. Science 312:1389-1392. https://doi.org/10.1126/science.1123511

8. Braak H, Brettschneider J, Ludolph AC, Lee VM, Trojanowski JQ, Del Tredici K (2013) Amyotrophic lateral sclerosis--a model of corticofugal axonal spread. Nat Rev Neurol 9:708-714. https://doi.org/10.1038/nrneurol.2013.221

9. Braak H, Ludolph AC, Neumann M, Ravits J, Del Tredici K (2017) Pathological TDP-43 changes in Betz cells differ from those in bulbar and spinal alphamotoneurons in sporadic amyotrophic lateral sclerosis. Acta Neuropathol 133:79-90. https://doi.org/10.1007/s00401-016-1633-2

10. Bradl M, Lassmann H (2010) Oligodendrocytes: biology and pathology. Acta Neuropathol 119:37-53. https://doi.org/10.1007/s00401-009-0601-5

11. Brettschneider J, Del Tredici K, Toledo JB, Robinson JL, Irwin DJ, Grossman M, Suh E, Van Deerlin VM, Wood EM, Baek Y et al (2013) Stages of pTDP-43 pathology in amyotrophic lateral sclerosis. Ann Neurol 74:20-38. https://doi. org/10.1002/ana.23937

12. Brettschneider J, Libon DJ, Toledo JB, Xie SX, McCluskey L, Elman L, Geser F, Lee VM, Grossman M, Trojanowski JQ (2012) Microglial activation and TDP-43 pathology correlate with executive dysfunction in amyotrophic lateral sclerosis. Acta Neuropathol 123:395-407. https://doi.org/10.1007/s00401-011-0932-x

13. Brockington A, Ning K, Heath PR, Wood E, Kirby J, Fusi N, Lawrence N, Wharton SB, Ince PG, Shaw PJ (2013) Unravelling the enigma of selective vulnerability in neurodegeneration: motor neurons resistant to degeneration in ALS show distinct gene expression characteristics and decreased susceptibility to excitotoxicity. Acta Neuropathol 125:95-109. https://doi.org/10.1007/s00401-012-1058-5

14. Cairns NJ, Neumann M, Bigio EH, Holm IE, Troost D, Hatanpaa KJ, Foong C, White CL 3rd, Schneider JA, Kretzschmar HA et al (2007) TDP-43 in familial and sporadic frontotemporal lobar degeneration with ubiquitin inclusions. Am J Pathol 171:227-240. https://doi.org/10.2353/ajpath.2007. 070182

15. Can A, Gerdes MJ, Tao X, Bello MO, Seel M (2013) Method and apparatus for detecting irregularities in tissue microarrays. General Electric Co, City

16. Cowan CM, Mudher A (2013) Are tau aggregates toxic or protective in tauopathies? Front Neurol 4:114. https://doi.org/10.3389/fneur.2013.00114

17. DeJesus-Hernandez M, Mackenzie IR, Boeve BF, Boxer AL, Baker M, Rutherford NJ, Nicholson AM, Finch NA, Flynn H, Adamson J et al (2011) Expanded GGGGCC hexanucleotide repeat in noncoding region of C9ORF72 causes chromosome 9p-linked FTD and ALS. Neuron 72:245-256. https://doi.org/10.1016/j.neuron.2011.09.011

18. Deming Y, Li Z, Kapoor M, Harari O, Del-Aguila JL, Black K, Carrell D, Cai Y, Fernandez MV, Budde Jet al (2017) Genome-wide association study identifies four novel loci associated with Alzheimer's endophenotypes and disease modifiers. Acta Neuropathol 133: 839-856 Doi https://doi.org/10. 1007/s00401-017-1685-y

19. Eisen A, Kim S, Pant B (1992) Amyotrophic lateral sclerosis (ALS): a phylogenetic disease of the corticomotoneuron? Muscle Nerve 15:219-224. https://doi.org/10.1002/mus.880150215

20. Feiler MS, Strobel B, Freischmidt A, Helferich AM, Kappel J, Brewer BM, Li D, Thal DR, Walther P, Ludolph AC et al (2015) TDP-43 is intercellularly transmitted across axon terminals. J Cell Biol 211:897-911. https://doi.org/ 10.1083/jcb.201504057

21. Forsberg K, Andersen PM, Marklund SL, Brannstrom T (2011) Glial nuclear aggregates of superoxide dismutase-1 are regularly present in patients with amyotrophic lateral sclerosis. Acta Neuropathol 121:623-634. https://doi.org/ 10.1007/s00401-011-0805-3

22. Forsberg K, Graffmo K, Pakkenberg B, Weber M, Nielsen M, Marklund S, Brannstrom T, Andersen PM (2019) Misfolded SOD1 inclusions in patients with mutations in C9orf72 and other ALS/FTD-associated genes. J Neurol Neurosurg Psychiatry 90:861-869. https://doi.org/10.1136/jnnp-2018-319386

23. Gal J, Strom AL, Kilty R, Zhang F, Zhu H (2007) p62 accumulates and enhances aggregate formation in model systems of familial amyotrophic lateral sclerosis. J Biol Chem 282:11068-11077. https://doi.org/10.1074/jbc. M608787200

24. Geevasinga N, Menon P, Ozdinler PH, Kiernan MC, Vucic S (2016) Pathophysiological and diagnostic implications of cortical dysfunction in ALS. Nat Rev Neurol 12:651-661. https://doi.org/10.1038/nrneurol.2016.140

25. Gerdes MJ, Sevinsky CJ, Sood A, Adak S, Bello MO, Bordwell A, Can A, Corwin A, Dinn S, Filkins RJ et al (2013) Highly multiplexed single-cell analysis of formalin-fixed, paraffin-embedded cancer tissue. Proc Natl Acad Sci U S A 110:11982-11987. https://doi.org/10.1073/pnas.1300136110

26. Giannini LAA, Xie SX, Peterson C, Zhou C, Lee EB, Wolk DA, Grossman M, Trojanowski JQ, McMillan CT, Irwin DJ (2019) Empiric methods to account for pre-analytical variability in digital histopathology in Frontotemporal lobar degeneration. Front Neurosci 13:682. https://doi.org/10.3389/fnins. 2019.00682

27. Grothe MJ, Sepulcre J, Gonzalez-Escamilla G, Jelistratova I, Scholl M, Hansson O, Teipel SJ, Alzheimer's Disease Neuroimaging I (2018) Molecular properties underlying regional vulnerability to Alzheimer's disease pathology. Brain 141:2755-2771. https://doi.org/10.1093/brain/awy189

28. Guo T, Noble W, Hanger DP (2017) Roles of tau protein in health and disease. Acta Neuropathol 133:665-704. https://doi.org/10.1007/s00401-017-1707-9

29. Hara M, Minami M, Kamei S, Suzuki N, Kato M, Aoki M (2012) Lower motor neuron disease caused by a novel FUS/TLS gene frameshift mutation. J Neurol 259:2237-2239. https://doi.org/10.1007/s00415-012-6542-2

30. Ince P, Stout N, Shaw P, Slade J, Hunziker W, Heizmann CW, Baimbridge KG (1993) Parvalbumin and calbindin D-28k in the human motor system and in motor neuron disease. Neuropathol Appl Neurobiol 19:291-299

31. Ince PG, Tomkins J, Slade JY, Thatcher NM, Shaw PJ (1998) Amyotrophic lateral sclerosis associated with genetic abnormalities in the gene encoding $\mathrm{cu} / \mathrm{Zn}$ superoxide dismutase: molecular pathology of five new cases, and comparison with previous reports and 73 sporadic cases of ALS.J Neuropathol Exp Neurol 57:895-904. https://doi.org/10.1097/00005072199810000-00002 
32. Ito Y, Ofengeim D, Najafov A, Das S, Saberi S, Li Y, Hitomi J, Zhu H, Chen H, Mayo L et al (2016) RIPK1 mediates axonal degeneration by promoting inflammation and necroptosis in ALS. Science 353:603-608. https://doi.org/ 10.1126/science.aaf6803

33. Jara JH, Genc B, Stanford MJ, Pytel P, Roos RP, Weintraub S, Mesulam MM, Bigio EH, Miller RJ, Ozdinler PH (2017) Evidence for an early innate immune response in the motor cortex of ALS. J Neuroinflammation 14:129. https:// doi.org/10.1186/s12974-017-0896-4

34. Johnson BS, Snead D, Lee JJ, McCaffery JM, Shorter J, Gitler AD (2009) TDP43 is intrinsically aggregation-prone, and amyotrophic lateral sclerosis-linked mutations accelerate aggregation and increase toxicity. J Biol Chem 284: 20329-20339. https://doi.org/10.1074/jbc.M109.010264

35. Josephs KA, Whitwell JL, Weigand SD, Murray ME, Tosakulwong N, Liesinger AM, Petrucelli L, Senjem ML, Knopman DS, Boeve BF et al (2014) TDP-43 is a key player in the clinical features associated with Alzheimer's disease. Acta Neuropathol 127:811-824. https://doi.org/10.1007/s00401-014-1269-z

36. Kang SH, Li Y, Fukaya M, Lorenzini I, Cleveland DW, Ostrow LW, Rothstein JD, Bergles DE (2013) Degeneration and impaired regeneration of gray matter oligodendrocytes in amyotrophic lateral sclerosis. Nat Neurosci 16: 571-579. https://doi.org/10.1038/nn.3357

37. Kim HJ, Raphael AR, LaDow ES, McGurk L, Weber RA, Trojanowski JQ, Lee VMY, Finkbeiner S, Gitler AD, Bonini NM (2014) Therapeutic modulation of elF2 alpha phosphorylation rescues TDP-43 toxicity in amyotrophic lateral sclerosis disease models. Nat Genet 46:152. https://doi.org/10.1038/ng.2853

38. Kim J, Hughes EG, Shetty AS, Arlotta P, Goff LA, Bergles DE, Brown SP (2017) Changes in the excitability of neocortical neurons in a mouse model of amyotrophic lateral sclerosis are not specific to Corticospinal neurons and are modulated by advancing Disease. J Neurosci 37:9037-9053. https://doi. org/10.1523/JNEUROSCI.0811-17.2017

39. King A, Bodi I, Nolan M, Troakes C, Al-Sarraj S (2015) Assessment of the degree of asymmetry of pathological features in neurodegenerative diseases. What is the significance for brain banks? J Neural Transm (Vienna) 122:1499-1508. https://doi.org/10.1007/s00702-015-1410-8

40. King A, Troakes C, Smith B, Nolan M, Curran O, Vance C, Shaw CE, Al-Sarraj S (2015) ALS-FUS pathology revisited: singleton FUS mutations and an unusual case with both a FUS and TARDBP mutation. Acta Neuropathol Commun 3:62. https://doi.org/10.1186/s40478-015-0235-x

41. Kushner PD, Stephenson DT, Wright S (1991) Reactive astrogliosis is widespread in the subcortical white matter of amyotrophic lateral sclerosis brain. J Neuropathol Exp Neurol 50:263-277

42. Kuusisto E, Kauppinen T, Alafuzoff I (2008) Use of p62/SQSTM1 antibodies for neuropathological diagnosis. Neuropathol Appl Neurobiol 34:169-180. https://doi.org/10.1111/j.1365-2990.2007.00884.x

43. Kwiatkowski TJ Jr, BosCo DA, Leclerc AL, Tamrazian E, Vanderburg CR, Russ C, Davis A, Gilchrist J, Kasarskis EJ, Munsat T et al (2009) Mutations in the FUS/TLS gene on chromosome 16 cause familial amyotrophic lateral sclerosis. Science 323:1205-1208. https://doi.org/10.1126/science.1166066

44. Lee SE, Lee Y, Lee GH (2019) The regulation of glutamic acid decarboxylases in GABA neurotransmission in the brain. Arch Pharm Res 42:1031-1039. https://doi.org/10.1007/s12272-019-01196-z

45. Liu G, Coyne AN, Pei F, Vaughan S, Chaung M, Zarnescu DC, Buchan JR (2017) Endocytosis regulates TDP-43 toxicity and turnover. Nat Commun 8: 2092. https://doi.org/10.1038/s41467-017-02017-x

46. Mackenzie IR, Ansorge O, Strong M, Bilbao J, Zinman L, Ang LC, Baker M, Stewart H, Eisen A, Rademakers R et al (2011) Pathological heterogeneity in amyotrophic lateral sclerosis with FUS mutations: two distinct patterns correlating with disease severity and mutation. Acta Neuropathol 122:87-98. https://doi.org/10.1007/s00401-011-0838-7

47. Mackenzie IR, Neumann M, Baborie A, Sampathu DM, Du Plessis D, Jaros E, Perry RH, Trojanowski JQ, Mann DM, Lee VM (2011) A harmonized classification system for FTLD-TDP pathology. Acta Neuropathol 122:111113. https://doi.org/10.1007/s00401-011-0845-8

48. Maekawa S, Al-Sarraj S, Kibble M, Landau S, Parnavelas J, Cotter D, Everall I, Leigh PN (2004) Cortical selective vulnerability in motor neuron disease: a morphometric study. Brain 127:1237-1251. https:/doi.org/10.1093/brain/awh132

49. McCauley ME, Baloh RH (2019) Inflammation in ALS/FTD pathogenesis. Acta Neuropathol 137:715-730. https://doi.org/10.1007/s00401-018-1933-9

50. Molyneaux BJ, Arlotta P, Menezes JR, Macklis JD (2007) Neuronal subtype specification in the cerebral cortex. Nat Rev Neurosci 8:427-437. https://doi. org/10.1038/nrn2151
51. Munoz DG, Neumann M, Kusaka H, Yokota O, Ishihara K, Terada S, Kuroda S, Mackenzie IR (2009) FUS pathology in basophilic inclusion body disease. Acta Neuropathol 118:617-627. https://doi.org/10.1007/s00401-009-0598-9

52. Nelder JA, Wedderburn WM (1972) Generalized linear models. J R Stat Soc A 135:15. https://doi.org/10.2307/2344614

53. Nelson PT, Estus S, Abner EL, Parikh I, Malik M, Neltner JH, Ighodaro E, Wang WX, Wilfred BR, Wang LS et al (2014) ABCC9 gene polymorphism is associated with hippocampal sclerosis of aging pathology. Acta Neuropathol 127:825-843. https://doi.org/10.1007/s00401-014-1282-2

54. Neumann M, Sampathu DM, Kwong LK, Truax AC, Micsenyi MC, Chou TT, Bruce J, Schuck T, Grossman M, Clark CM et al (2006) Ubiquitinated TDP-43 in frontotemporal lobar degeneration and amyotrophic lateral sclerosis. Science 314:130-133. https://doi.org/10.1126/science.1134108

55. Nihei K, McKee AC, Kowall NW (1993) Patterns of neuronal degeneration in the motor cortex of amyotrophic lateral sclerosis patients. Acta Neuropathol 86:55-64

56. Nijssen J, Comley LH, Hedlund E (2017) Motor neuron vulnerability and resistance in amyotrophic lateral sclerosis. Acta Neuropathol 133:863-885. https://doi.org/10.1007/s00401-017-1708-8

57. O'Rourke JG, Bogdanik L, Yanez A, Lall D, Wolf AJ, Muhammad AK, Ho R, Carmona S, Vit JP, Zarrow J et al (2016) C9orf72 is required for proper macrophage and microglial function in mice. Science 351:1324-1329. https://doi.org/10.1126/science.aaf1064

58. Pamphlett R, Kum Jew S (2008) TDP-43 inclusions do not protect motor neurons from sporadic ALS. Acta Neuropathol 116:221-222. https://doi.org/ 10.1007/s00401-008-0392-0

59. Paolicelli RC, Jawaid A, Henstridge CM, Valeri A, Merlini M, Robinson JL, Lee EB, Rose J, Appel S, Lee VM et al (2017) TDP-43 depletion in microglia promotes amyloid clearance but also induces synapse loss. Neuron 95(297308):e296. https://doi.org/10.1016/..neuron.2017.05.037

60. Parkinson N, Ince PG, Smith MO, Highley R, Skibinski G, Andersen PM, Morrison KE, Pall HS, Hardiman O, Collinge J et al (2006) ALS phenotypes with mutations in CHMP2B (charged multivesicular body protein 2B). Neurology 67:1074-1077. https://doi.org/10.1212/01.wnl.0000231510.89311.8b

61. Philips T, Bento-Abreu A, Nonneman A, Haeck W, Staats K, Geelen V, Hersmus N, Kusters B, Van Den Bosch L, Van Damme P et al (2013) Oligodendrocyte dysfunction in the pathogenesis of amyotrophic lateral sclerosis. Brain 136:471-482. https://doi.org/10.1093/brain/aws339

62. Philips T, Robberecht W (2011) Neuroinflammation in amyotrophic lateral sclerosis: role of glial activation in motor neuron disease. Lancet Neurol 10: 253-263. https://doi.org/10.1016/S1474-4422(11)70015-1

63. Porta $\mathrm{S}, \mathrm{Xu} Y$, Restrepo CR, Kwong LK, Zhang B, Brown HJ, Lee EB, Trojanowski $J Q$, Lee VM (2018) Patient-derived frontotemporal lobar degeneration brain extracts induce formation and spreading of TDP-43 pathology in vivo. Nat Commun 9:4220. https://doi.org/10.1038/s41467-018-06548-9

64. Renton AE, Majounie E, Waite A, Simon-Sanchez J, Rollinson S, Gibbs JR, Schymick JC, Laaksovirta H, van Swieten JC, Myllykangas L et al (2011) A hexanucleotide repeat expansion in C9ORF72 is the cause of chromosome 9p21linked ALS-FTD. Neuron 72:257-268. https:/doi.org/10.1016/j.neuron.2011.09.010

65. Restagno G, Lombardo F, Sbaiz L, Mari C, Gellera C, Alimonti D, Calvo A, Tarenzi L, Chio A (2008) The rare G93D mutation causes a slowly progressing lower motor neuron disease. Amyotroph Lateral Scler 9:35-39. https://doi.org/10.1080/17482960701788198

66. Rizzu P, Blauwendraat C, Heetveld S, Lynes EM, Castillo-Lizardo M, Dhingra A, Pyz E, Hobert M, Synofzik M, Simon-Sanchez J et al (2016) C9orf72 is differentially expressed in the central nervous system and myeloid cells and consistently reduced in C9orf72, MAPT and GRN mutation carriers. Acta Neuropathol Commun 4:37. https://doi.org/10.1186/s40478-016-0306-7

67. Rohan Z, Matej R, Rusina R, Kovacs GG (2014) Oligodendroglial response in the spinal cord in TDP-43 proteinopathy with motor neuron involvement. Neurodegener Dis 14:117-124. https://doi.org/10.1159/000362929

68. Rosen DR, Siddique T, Patterson D, Figlewicz DA, Sapp P, Hentati A, Donaldson D, Goto J, O'Regan JP, Deng HX et al (1993) Mutations in Cu/Zn superoxide dismutase gene are associated with familial amyotrophic lateral sclerosis. Nature 362:59-62. https://doi.org/10.1038/362059a0

69. Rudy B, Fishell G, Lee S, Hjerling-Leffler J (2011) Three groups of interneurons account for nearly $100 \%$ of neocortical GABAergic neurons. Dev Neurobiol 71:45-61. https://doi.org/10.1002/dneu.20853

70. Sasaki S, Iwata M (2000) Immunocytochemical and ultrastructural study of the motor cortex in patients with lower motor neuron disease. Neurosci Lett 281:45-48 
71. Schneider CA, Rasband WS, Eliceiri KW (2012) NIH image to ImageJ: 25 years of image analysis. Nat Methods 9:671-675

72. Schwab C, Yu S, Wong W, McGeer EG, McGeer PL (2013) GAD65, GAD67, and GABAT immunostaining in human brain and apparent GAD65 loss in Alzheimer's disease. J Alzheimers Dis 33:1073-1088. https://doi.org/10.3233/ JAD-2012-121330

73. Sidhu A, Wersinger C, Moussa CE, Vernier P (2004) The role of alphasynuclein in both neuroprotection and neurodegeneration. Ann N Y Acad Sci 1035:250-270. https://doi.org/10.1196/annals.1332.016

74. Sitte HH, Wanschitz J, Budka H, Berger ML (2001) Autoradiography with [3H]PK11195 of spinal tract degeneration in amyotrophic lateral sclerosis. Acta Neuropathol 101:75-78

75. Stefanits H, Budka H, Kovacs GG (2012) Asymmetry of neurodegenerative disease-related pathologies: a cautionary note. Acta Neuropathol 123:449452. https://doi.org/10.1007/s00401-011-0936-6

76. Suzuki N, Kato S, Kato M, Warita H, Mizuno H, Kato M, Shimakura N, Akiyama H, Kobayashi Z, Konno H et al (2012) FUS/TLS-immunoreactive neuronal and glial cell inclusions increase with disease duration in familial amyotrophic lateral sclerosis with an R521C FUS/TLS mutation. J Neuropathol Exp Neurol 71:779-788. https://doi.org/10.1097/NEN.0b013e318264f164

77. Takeuchi R, Tada M, Shiga A, Toyoshima Y, Konno T, Sato T, Nozaki H, Kato T. Horie M, Shimizu H et al (2016) Heterogeneity of cerebral TDP-43 pathology in sporadic amyotrophic lateral sclerosis: evidence for clinicopathologic subtypes. Acta Neuropathol Commun 4:61. https://doi.org/10. 1186/s40478-016-0335-2

78. Tan RH, Yang Y, Kim WS, Dobson-Stone C, Kwok JB, Kiernan MC, Halliday GM (2017) Distinct TDP-43 inclusion morphologies in frontotemporal lobar degeneration with and without amyotrophic lateral sclerosis. Acta Neuropathol Commun 5:76. https://doi.org/10.1186/s40478-017-0480-2

79. Tumer Z, Bertelsen B, Gredal O, Magyari M, Nielsen KC, Lucamp GK, Brondum-Nielsen K (2012) Novel heterozygous nonsense mutation of the OPTN gene segregating in a Danish family with ALS. Neurobiol Aging 33: 208 e201-208 e205. https://doi.org/10.1016/.neurobiolaging.2011.07.001

80. Turner MR, Cagnin A, Turkheimer FE, Miller CC, Shaw CE, Brooks DJ, Leigh PN, Banati RB (2004) Evidence of widespread cerebral microglial activation in amyotrophic lateral sclerosis: an [11C](R)-PK11195 positron emission tomography study. Neurobiol Dis 15:601-609. https://doi.org/10.1016/.jnbd.2003.12.012

81. Van Deerlin VM, Leverenz JB, Bekris LM, Bird TD, Yuan W, Elman LB, Clay D, Wood EM, Chen-Plotkin AS, Martinez-Lage M et al (2008) TARDBP mutations in amyotrophic lateral sclerosis with TDP-43 neuropathology: a genetic and histopathological analysis. Lancet Neurol 7:409-416. https://doi.org/10.1016/ S1474-4422(08)70071-1

82. Walker AK, Spiller KJ, Ge G, Zheng A, Xu Y, Zhou M, Tripathy K, Kwong LK, Trojanowski JQ, Lee VM (2015) Functional recovery in new mouse models of ALS/FTLD after clearance of pathological cytoplasmic TDP-43. Acta Neuropathol 130:643-660. https://doi.org/10.1007/s00401-015-1460-x

83. Wolfinger R, O'Connell M (1993) Generalized linear mixed models: a pseudo-likelihood approach. J Stat Comput Simul 48:10

84. Woolfe F, Gerdes M, Bello M, Tao X, Can A (2011) Autofluorescence removal by non-negative matrix factorization. IEEE Trans Image Process 20:10851093. https://doi.org/10.1109/TIP.2010.2079810

85. Yang Y, Halliday GM, Kiernan MC, Tan RH (2019) TDP-43 levels in the brain tissue of ALS cases with and without C9ORF72 or ATXN2 gene expansions. Neurology 93:e1748-e1755. https://doi.org/10.1212/WNL.0000000000008439

86. Yousef A, Robinson JL, Irwin DJ, Byrne MD, Kwong LK, Lee EB, Xu Y, Xie SX, Rennert L, Suh E et al (2017) Neuron loss and degeneration in the progression of TDP-43 in frontotemporal lobar degeneration. Acta Neuropathol Commun 5:68. https://doi.org/10.1186/s40478-017-0471-3

87. Zhang W, Zhang L, Liang B, Schroeder D, Zhang ZW, Cox GA, Li Y, Lin DT (2016) Hyperactive somatostatin interneurons contribute to excitotoxicity in neurodegenerative disorders. Nat Neurosci 19:557-559. https://doi.org/10. 1038/nn.4257

\section{Publisher's Note}

Springer Nature remains neutral with regard to jurisdictional claims in published maps and institutional affiliations.

\section{Ready to submit your research? Choose BMC and benefit from:}

- fast, convenient online submission

- thorough peer review by experienced researchers in your field

- rapid publication on acceptance

- support for research data, including large and complex data types

- gold Open Access which fosters wider collaboration and increased citations

- maximum visibility for your research: over $100 \mathrm{M}$ website views per year

At BMC, research is always in progress.

Learn more biomedcentral.com/submissions 